\title{
Floor plate-derived neuropilin-2 functions as a secreted semaphorin sink to facilitate commissural axon midline crossing
}

\author{
Berenice Hernandez-Enriquez, ${ }^{1,6}$ Zhuhao Wu, ${ }^{2,6}$ Edward Martinez, ${ }^{1}$ Olav Olsen, ${ }^{2}$ Zaven Kaprielian, ${ }^{3}$ \\ Patricia F. Maness, ${ }^{4}$ Yutaka Yoshida, ${ }^{5}$ Marc Tessier-Lavigne, ${ }^{2}$ and Tracy S. Tran ${ }^{1}$ \\ ${ }^{1}$ Department of Biological Sciences, Rutgers University, Newark, New Jersey 07102, USA; ${ }^{2}$ Laboratory of Brain Development \\ and Repair, The Rockefeller University, New York, New York 10065, USA; ${ }^{3}$ Amgen, Cambridge, Massachusetts 02141, USA; \\ ${ }^{4}$ Department of Biochemistry and Biophysics, University of North Carolina School of Medicine, Chapel Hill, North \\ Carolina 27599, USA; ${ }^{5}$ Division of Developmental Biology, Cincinnati Children's Hospital Medical Center, Cincinnati, \\ Ohio 45229, USA
}

Commissural axon guidance depends on a myriad of cues expressed by intermediate targets. Secreted semaphorins signal through neuropilin-2/plexin-A1 receptor complexes on post-crossing commissural axons to mediate floor plate repulsion in the mouse spinal cord. Here, we show that neuropilin-2/plexin-A1 are also coexpressed on commissural axons prior to midline crossing and can mediate precrossing semaphorin-induced repulsion in vitro. How premature semaphorin-induced repulsion of precrossing axons is suppressed in vivo is not known. We discovered that a novel source of floor plate-derived, but not axon-derived, neuropilin-2 is required for precrossing axon pathfinding. Floor plate-specific deletion of neuropilin-2 significantly reduces the presence of precrossing axons in the ventral spinal cord, which can be rescued by inhibiting plexin-A1 signaling in vivo. Our results show that floor platederived neuropilin-2 is developmentally regulated, functioning as a molecular sink to sequester semaphorins, preventing premature repulsion of precrossing axons prior to subsequent down-regulation, and allowing for semaphorin-mediated repulsion of post-crossing axons.

[Keywords: spinal cord; pathfinding; semaphorin 3B; explant cultures; neural development]

Supplemental material is available for this article.

Received June 27, 2015; revised version accepted November 13, 2015.

In bilaterally symmetric organisms, commissural neurons and their axons play a key role in transferring information from one side of the central nervous system (CNS) to the other (Kaprielian et al. 2001; Vallstedt and Kullander 2013). The highly stereotyped trajectories of commissural axon projections allow them to serve as a model system for investigating mechanisms of axon guidance during neural development (Kolodkin and Tessier-Lavigne 2011). Spinal cord commissural axons are attracted to the CNS midline by floor plate-derived chemoattractants, such as netrin, while floor plate-derived repellents, including semaphorins, expel axons from the floor plate and prevent their recrossing (Kolodkin and Tessier-Lavigne 2011). Previous studies have shown that a number of class 3 secreted semaphorins (Sema3s) are expressed in the developing mammalian spinal cord (Zou et al.

${ }^{6}$ These authors contributed equally to this work.

Corresponding author: tstran@rutgers.edu

Article is online at http://www.genesdev.org/cgi/doi/10.1101/gad.268086. 115 .
2000; Cohen et al. 2005; Huber et al. 2005). Notably, Sema3B is highly expressed at the midline by floor plate cells, while Sema3F is more broadly expressed in the intermediate zone (Zou et al. 2000). In vitro assays using rodent spinal cord explants demonstrate that both Sema3B and Sema3F strongly inhibit the growth of commissural axons that have crossed the floor plate (post-crossing) but do not affect the growth of axons that have not encountered the floor plate (precrossing) (Zou et al. 2000), suggesting a post-crossing function for Sema3 signaling in commissural axon guidance.

Neuropilin-2 (Nrp2) is an obligate coreceptor for Sema3s (Chen et al. 1997; Giger et al. 1998) and regulates a number of neurodevelopmental processes (Tran et al. 2007; Yoshida 2012; Jongbloets and Pasterkamp 2014).

(C) 2015 Hernandez-Enriquez et al. This article is distributed exclusively by Cold Spring Harbor Laboratory Press for the first six months after the full-issue publication date (see http://genesdev.cshlp.org/site/misc/ terms.xhtml). After six months, it is available under a Creative Commons License (Attribution-NonCommercial 4.0 International), as described at http://creativecommons.org/licenses/by-nc/4.0/. 
These include the guidance of axons in select central and peripheral axonal tracks and nerves (Chen et al. 1998, 2000; Giger et al. 2000; Cloutier et al. 2002; Walz et al. 2002; Huber et al. 2005; Claudepierre et al. 2008; Kolk et al. 2009; Demyanenko et al. 2011). Previous work has shown that Nrp2 mRNA is prominently expressed in neurons that extend commissural axons (Zou et al. 2000; Nawabi et al. 2010). Nrp2 protein expression is in line with this mRNA expression profile; robust Nrp2 protein levels are observed within a subpopulation of dorsal spinal commissural neurons that are positive for the Atoh-1/Math-1 transcription factor and also in a subset of ventral GABAergic commissural neurons (Phelps et al. 1999; Tran et al. 2013). Nrp2-deficient mice display various spinal commissural axon guidance defects during midline crossing at the floor plate and in post-crossing axon trajectories (Zou et al. 2000; Tran et al. 2013); however, Nrp2 mutants do not exhibit any commissural axon precrossing guidance errors.

Plexin-A1 (PlxnA1) forms a holoreceptor complex with neuropilins (Rohm et al. 2000) to signal Sema3-mediated repulsion of commissural axons (Mann and Rougon 2007). Interestingly, Nrp2-positive precrossing commissural axons are insensitive to Sema3B, and this is thought to be due to proteolytic processing of PlxnA1 receptors, a process that is down-regulated in commissural axons when they encounter NrCAM, a cell adhesion molecule, in the floor plate (Nawabi et al. 2010). However, we observed here that both PlxnA1 and Nrp2 proteins are expressed in precrossing axon segments in overlapping subsets of dorsal and ventral commissural axons in vivo. This unexpected finding led us to revisit the role played by Sema3 signaling in the guidance of precrossing commissural axons and ask the following questions: (1) Are Nrp2-positive precrossing axons sensitive to the repulsive effects of Sema3s in the developing mouse spinal cord? (2) If so, then what is the mechanism that prevents premature Sema3B-mediated repulsion of Nrp2-positive precrossing axons, allowing them to reach the ventral midline?

Here, we focus on the function of Nrp2 and PlxnA1 in both dorsal and ventral precrossing commissural axons, using in vivo mouse genetic experiments to investigate the functional role of a novel source of Nrp2 protein expressed by floor plate cells. We found that both Nrp2 and PlxnA1 expressed in dorsal and ventral commissural neurons are dispensable for precrossing commissural axon pathfinding. However, specific deletion of Nrp2 in floor plate cells causes a significant defect in the trajectories of these precrossing axons. Furthermore, we show that precrossing axons from dorsal spinal cord explants are indeed sensitive to Sema3B, but not Sema3F, and that precrossing axon guidance defects observed in mutant embryos that lack Nrp2 expression in the floor plate are rescued by inhibiting PlxnAl signaling in vivo. Taken together, our results demonstrate that select subsets of dorsal and ventral precrossing commissural axons are responsive to the inhibitory effects of Sema3B, but premature repulsion of precrossing axons is prevented by a novel, non-cell-autonomous function of Nrp2 in floor plate cells.

\section{Results}

Nrp2 and PlxnA1 are expressed in overlapping subsets of precrossing commissural axons

$N r p 2^{-/-}$embryos have defects in post-crossing commissural axon trajectories (Zou et al. 2000; Tran et al. 2013), consistent with the expression of Nrp2 protein in dorsal Atoh1/Math1-positive and ventral GABAergic neurons and their axons (Phelps et al. 1999; Tran et al. 2013). PlxnA1 mRNA is also detected in dorsal commissural neurons (Nawabi et al. 2010), but whether PlxnA1 protein is coexpressed with Nrp2 in commissural axon bundles is not known. Using a polyclonal rabbit PlxnA1 antibody raised against a peptide epitope from the most $\mathrm{C}$-terminal 16 amino acids of mouse PlxnA1 (Yoshida et al. 2006), we found that PlxnA1 colocalizes with Nrp2 in post-crossing segments of commissural axons (Fig. 1A, $\mathrm{A}^{\prime}$ ). However, PlxnAl was also prominently detected in precrossing segments, which was unexpected because a previous study showed that levels of PlxnA1 are low in commissural axons prior to encountering the floor plate (Nawabi et al. 2010). This result prompted us to test the specificity of our mouse PlxnAl antibody and the human PlxnAl antibody, used in the earlier study, on $P l \times n A 1^{-/-}$spinal cord sections. While both antibodies stained pre- and postcrossing commissural axons (Fig. 1A, $\mathrm{A}^{\prime}$; Supplemental Fig. 1A), only the mouse PlxnA1 staining was specifically lost in PlxnA1 ${ }^{-/-}$spinal cord sections (Supplemental Fig. 1B). A significant nonspecific immunofluorescent signal remained in $P \operatorname{lxnA1^{-/-}}$ tissue stained with the human PlxnA1 antibody (Supplemental Fig. 1A). Colocalization of Nrp2 and PlxnA1 with a precrossing commissural axon marker, transient axonal glycoprotein 1 (TAG1), confirmed that PlxnA1 and Nrp2 are expressed in precrossing segments within a subset of commissural axons (Fig. 1A,A'; Supplement Fig. 1), which can be detected as early as embryonic day 10.5 (E10.5) (Fig. 1B) until at least E12.5 (Fig. 1C).

Previous work has suggested that premature semaphorin-mediated repulsion of precrossing axons is prevented through the active degradation of PlxnAl by calpain1 in precrossing axons (Nawabi et al. 2010). The model also suggested that interaction of commissural axons with NrCAM at the floor plate suppresses PlxnA1 cleavage, thereby promoting up-regulation of PlxnA1 in post-crossing axons. However, we found that levels of PlxnA1 in precrossing, crossing, and post-crossing axons of wild-type embryos were similar to the levels in $N r C A M^{-/-}$mutant littermates. Furthermore, colocalization of Nrp2 with PlxnA1 in precrossing axons (Fig. 1D) suggests that PlxnA1 and Nrp2 may form functional semaphorin receptors in precrossing commissural axons.

\section{Sema3B inhibits precrossing commissural axon outgrowth}

Coexpression of Nrp2 and PlxnA1 in precrossing axons suggests that commissural axons may respond to Sema3s prior to midline crossing. To test this idea, E11 dorsal 


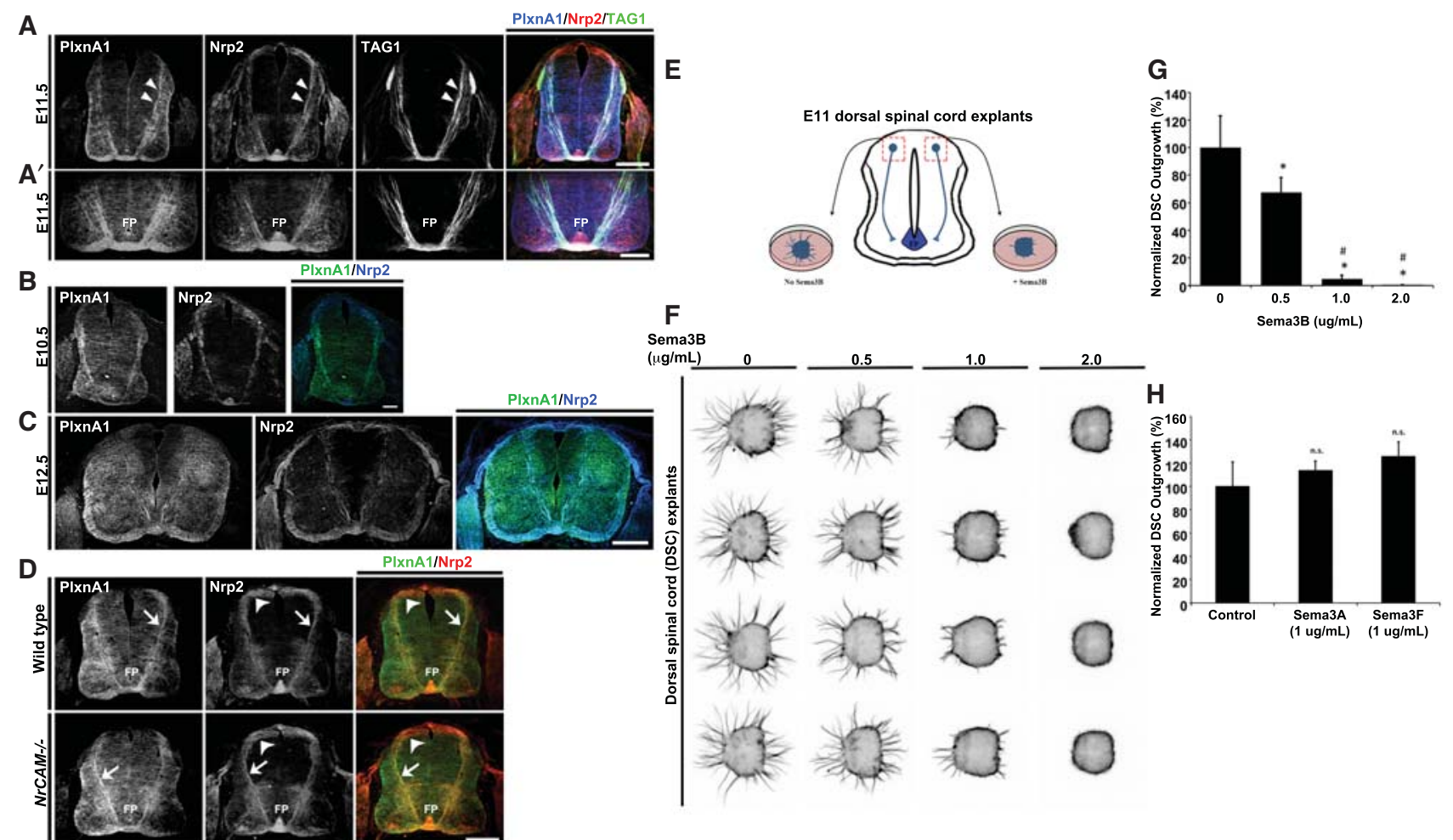

Figure 1. Dorsal commissural precrossing neurons express Nrp2 and PlxnA1, and their axons are responsive to Sema3B inhibition in a dosage-dependent manner. (A) Representative confocal images of a transverse wild-type mouse spinal cord section at E11.5 labeled with antibodies against PlxnA1 (blue) (Yoshida et al. 2006), Nrp2 (red), and TAG1 (green). White arrowheads illustrate the coexpression of PlxnA1, Nrp2, and TAG1 on precrossing axons in the dorsal spinal cord. Bar, $125 \mu \mathrm{m} .\left(A^{\prime}\right)$ Higher-magnification images of the same spinal cord section shown in $A$ illustrate colabeling of PlxnA1, Nrp2, and TAG1 in the ventral spinal cord and the ventral commissure. (FP) Floor plate. Bar, $75 \mu \mathrm{m}$. PlxnA1 antibody specificity is shown in Supplemental Figure 1. (B) Wild-type E10.5 spinal cord transverse section double labeled with antibodies against mouse PlxnA1 (left panel) and Nrp2 (middle panel) and merged (right panel). Bar, $60 \mu \mathrm{m}$. (C) Wild-type E12.5 spinal cord transverse section double labeled with antibodies against mouse PlxnA1 (left panel) and Nrp2 (middle panel) and merged (right panel). Bar, $250 \mu \mathrm{m}$. (D) E11.5 transverse spinal cord sections taken from wild-type and NrCAM-null animals and processed for immunolabeling against PlxnA1 and Nrp2. Dorsal commissural neuron cell bodies and their precrossing axons are labeled with white arrowheads and white arrows, respectively. Bar, $125 \mu \mathrm{m}$. (E) Schematic diagram illustrating the microdissection of E11 dorsal spinal cord explants for culture and analyzing precrossing axon outgrowth. $(F)$ Representative dorsal spinal cord explant cultures challenged with increasing levels of Sema3B proteins. (G) Axonal outgrowth from dorsal spinal explants were normalized and are shown in percentages with respect to the untreated $(100 \pm 11.45)$ cultures. A decrease of $>30 \%(67.54 \pm 5.29), 95 \%(4.97 \pm 1.22)$, and $100 \%(0.63 \pm 0.02)$ in outgrowth was observed with $0.5 \mu \mathrm{g} / \mathrm{mL}, 1.0 \mu \mathrm{g} / \mathrm{mL}$, and $2.0 \mu \mathrm{g} / \mathrm{mL}$ Sema3B, respectively. $(H)$ Normalized axonal outgrowth of dorsal commissural precrossing axons treated with Sema3A and Sema3F at $1 \mu \mathrm{g} / \mathrm{mL}$ concentration. The graph shows percentages normalized to untreated controls $(100 \pm 10.55)$, Sema3A (113.6 \pm 4.12$)$, and Sema3F $(125.6 \pm 6.29)$. Data represent compiled means \pm SEM from $n=4$ independent explant cultures. Analysis of variance (ANOVA) followed by post-hoc Tukey test, $\left({ }^{*}\right) P<0.05$ compared with $0 \mu \mathrm{g} / \mathrm{mL}$; (\#) $P<0.05$ compared with $0.5 \mu \mathrm{g} / \mathrm{mL}$ of Sema3B; (n.s.) not significant.

spinal cord explants containing the dorsal Nrp2-positive commissural neurons that have not yet extended their axons across the midline were cultured in the presence of Netrin1 for $1 \mathrm{~d}$ in vitro (Fig. 1E; Xu et al. 2014). Because Sema3B is the major Sema3 expressed by the floor plate (Zou et al. 2000; Nawabi et al. 2010), we challenged Netrin1-induced growth of precrossing axons with increasing levels of Sema3B. Sema3B $(0.5 \mu \mathrm{g} / \mathrm{mL})$ significantly inhibits axon growth from dorsal spinal cord explants, resulting in a $32 \%$ reduction in normalized axonal outgrowth compared with the controls (Fig. 1F,G). This response to Sema3B is dosage-dependent; axon outgrowth is inhibited $95 \%-100 \%$ in explants cultured with increasingly higher concentrations of Sema3B
(1 $\mu \mathrm{g} / \mathrm{mL}$ and $2 \mu \mathrm{g} / \mathrm{mL}$, respectively). Consistent with previous findings, we also found that Sema3B inhibits outgrowth of post-crossing axons in a dosage-dependent manner (Supplemental Fig. 2A,C). In contrast, neither Sema3A nor Sema3F significantly affect Netrin1-induced axon outgrowth (up to $1 \mu \mathrm{g} / \mathrm{mL}$ ) (Fig. $1 \mathrm{H}$ ), indicating that this semaphorin-induced effect on precrossing commissural axons is specific to Sema3B. Consistent with previous results, growth of post-crossing axons was inhibited by Sema3F but not Sema3A (Supplemental Fig. 2B,D). Taken together, these results indicate that precrossing axons are competent to sense Sema3B as they approach the floor plate and that post-crossing axons can rely on both Sema3B and Sema3F for their pathfinding. 
Nrp2 is expressed in the floor plate during precrossing commissural axon pathfinding

Sema3B is highly expressed in the floor plate at a time when commissural axons approach and cross the midline (Nawabi et al. 2010), suggesting that premature floor plate repulsion must be prevented in order for precrossing axons that respond to Sema3B to cross the midline. Immunolabeling of spinal cord sections for Nrp2 expression reveals a novel and unexpected source of Nrp2 at the ventral midline. Floor plate staining for Nrp2 is specific, as it is not present in the spinal cords of $N r p 2^{-/-}$embryos (Fig. 2). To determine whether the signal at the ventral midline originates from commissural axons crossing the midline or floor plate cells, we stained spinal cord sections with Nrp2 antibody and an antibody against a floor platespecific marker, BEN/ALCAM/SC1/DM-GRASP/MuSC
(Dillon et al. 2005). The majority of Nrp2 at the ventral midline colocalizes with BEN (Fig. 2A [see high-magnification images in bottom panels], B), suggesting that floor plate cells are a significant source of Nrp2 in the developing spinal cord. To extend this analysis, we detailed the developmental profile of Nrp2 expression in floor plate cells. Nrp2 is expressed in the floor plate as early as E9.5, a time before commissural axons even begin to project ventrally toward the midline (Fig. 2C). Interestingly, commissural axon expression of PlxnAl was first detected at E10.5 (Fig. 1B), $1 \mathrm{~d}$ later than the onset of floor plate-derived Nrp2 expression. The intensity of Nrp2 staining gradually increases between E9.5 and E11.5 and appears to peak at E11.5 (Fig. 2C). One day later, at E12.5, Nrp2 floor plate expression is dramatically reduced, and, by E13.5, Nrp2 expression is almost completely absent. The dynamic temporal regulation of Nrp2 floor plate
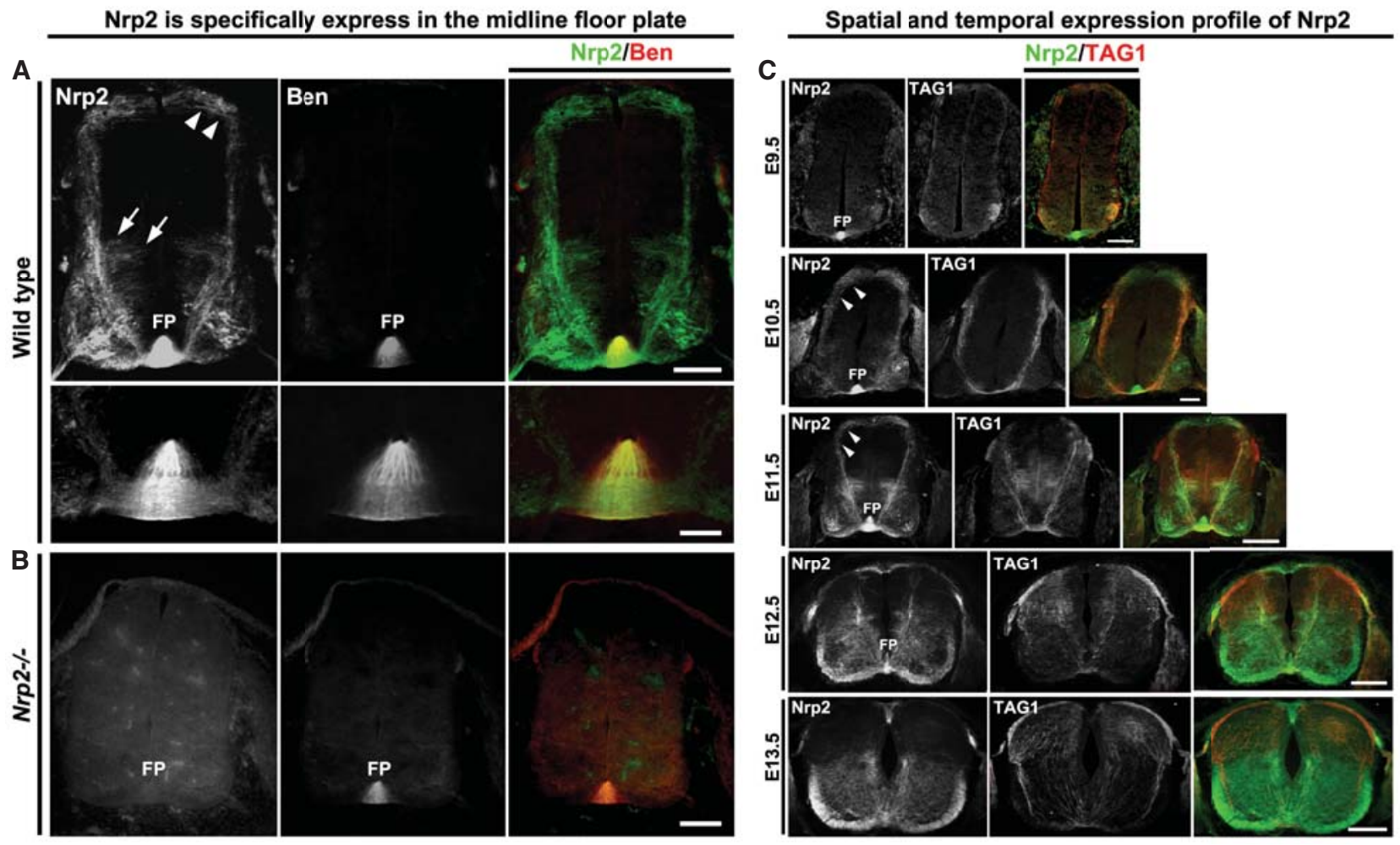

Figure 2. Nrp2 is highly expressed in the floor plate of the mouse spinal cord and is dynamically regulated during commissural axon pathfinding. (A) Wild-type E1 1.5 mouse spinal cord transverse sections show Nrp2 expression (green) in dorsal (white arrowheads) and ventral (white arrows) commissural neuron populations. Nrp2 is highly expressed in the floor plate and was colabeled with the floor plate-specific murine SC1-related protein (Ben; red). The bottom rows are a higher magnification of the top row images of the floor plate region. Bars: top row, $125 \mu \mathrm{m}$; bottom row, $25 \mu \mathrm{m}$. (B) A Nrp2 homozygote-null mutant (Nrp2 ${ }^{-/}$) spinal cord section at E11.5 colabeled with Nrp2 (green) and Ben (red). Bar, $125 \mu \mathrm{m}$. (C) Wild-type transverse spinal cord sections are shown colabeled with antibodies against Nrp2 (green) and TAG1 (red). Expression of Nrp2 in the floor plate can be detected as early as E9.5, at a developmental stage before commissural neurons extend axons toward the floor plate at the ventral midline. Bar, $100 \mu \mathrm{m}$. At E10.5, wild-type Nrp2-positive (green) commissural axons coexpressing TAG1 (red) have reached the ventral midline, where the floor plate robustly expresses Nrp2. Bar, $100 \mu \mathrm{m}$. At this stage, expression of PlxnA1 can also be detected in commissural axons, as shown in Figure 1C. Immunofluorescence of Nrp2 expression from both the midline crossing axons in ventral commissure and the floor plate cells is the highest at E11.5. Bar, $160 \mu \mathrm{m}$. While a few precrossing axons still express Nrp2 at E12.5, the floor plate-derived Nrp2 expression is down-regulated. Bar, $200 \mu \mathrm{m}$. By E13.5, the majority of commissural axons have crossed the midline, and the expression of Nrp2 from the floor plate has dramatically decreased in fluorescent intensity. Bar, $250 \mu \mathrm{m}$. White arrowheads point to dorsal commissural axons. (FP) Floor plate. 
expression coincides with the time when the majority of spinal commissural axons project toward the floor plate and cross the midline and begs the question of whether this robust source of Nrp2 at the floor plate plays a role in the guidance of precrossing commissural axons.

\section{Floor plate-derived Nrp2 is required for the guidance of precrossing axons of dorsal and ventral commissural neurons}

To examine whether floor plate-derived Nrp2 contributes to commissural axon guidance, conditional $N r p 2^{f / f}$ mice, which express GFP following Cre-mediated gene excision, were crossed to the FoxA2-CreERT2 line that drives cre recombinase expression specifically in floor plate cells in response to tamoxifen (TM) treatment (Park et al. 2008). Importantly, untreated E11.5 Nrp2 $2^{f / f}$; FoxA2 $2^{+/ C r e E R T 2}$ (no TM) embryos express Nrp2 in commissural axons and the floor plate at levels comparable with that of wild-type embryos (Figs. 1A, 3A). Following TM treatment, E11.5 Nrp2 $2^{f / f}{ }_{\text {F Fox } A 2^{+/ C r e E R T 2} \text { embryos }}$ show a specific loss of Nrp2 in floor plate cells without affecting Nrp2 expression in commissural axons (Fig. 3). Interestingly, floor plate-specific deletion of Nrp2 causes a misprojection of TAG1- and Nrp2-positive precrossing axons (Fig. 3A-C; Supplemental Fig. 3A,B). To confirm that floor plate-specific deletion of Nrp2 did not alter expression of Nrp2 and PlxnA1 in precrossing axons, open-book preparations of spinal cords from TM-treated and untreated E11.5 Nrp2 ${ }^{f / f}{ }_{j}$ FOXA2 $2^{+/ C r e E R T 2}$ animals were analyzed by immunofluorescent labeling of PlxnA1 and Nrp2. Commissural axon expression of PlxnA1 and Nrp2 was comparable between TM-treated Nrp2 $2^{f / f}$;FoxA2 $2^{+/ C r e E R T 2}$ and control animals (Supplemental Fig. 1C). Despite the weaker PlxnAl signal compared with Nrp2, there is measurable overlap of the two signals in precrossing axons (Supplemental Fig. 1C, white arrows). To quantify defects in the Nrp2 floor plate-specific deletion mutants, we systematically determined the specific immunofluorescence of Nrp2 and TAG1 in the ventral one-third of transverse spinal cord sections normalized to the size of each section measured (Fig. $3 \mathrm{D})$. The fluorescent intensity of Nrp2 and TAG1 is decreased by $>40 \%$ and $45 \%$, respectively, in $N r p 2^{f / f}$; FOXA2 ${ }^{+/ C r e E R T 2}+$ TM-treated (Nrp2, $7.52 \pm 0.50$; TAG1, $1.38 \pm 0.14)$ and $N r p 2^{f / f} ;$ FoxA2 $2^{\text {CreERT2/CreERT2 }}+$ TM-treated (Nrp2, 8.16 0.50; TAG1, $1.86 \pm 0.16)$ embryos compared with $\operatorname{Nrp}^{\text {flf }}{ }_{;}$FOXA $2^{+/ C r e E R T 2}$ with no TM (Nrp2, $11.89 \pm 0.72$; TAG1, 3.26 \pm 0.37 ) (Fig. 3E,G; see also Supplemental Table 1). We also determined the thickness of the ventral commissure, which was measured using the same criteria as previously described (Fig. 3D; Jaworski et al. 2010). The ventral commissure thickness using Nrp2 immunofluorescence was significantly reduced by $>25 \%$ and $50 \%$ in $N r p 2^{f / f}$;FoxA2 $2^{+/ C r e E R T 2}+\mathrm{TM}(0.0452 \pm$

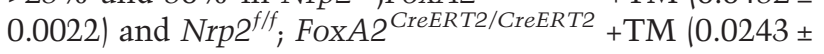
$0.0014)$ embryos, respectively, compared with untreated Nrp2 $2^{f / f}{ }_{;}$FoxA2 $2^{+/ C r e E R T 2}$ controls $(0.0614 \pm 0.0015)$ (Fig. 3F).

Precrossing defects were investigated further using whole-mount labeling of the spinal cords in the open- book preparation. Z-stack medial-to-lateral serial confocal images were acquired for viewing pre- and postcrossing axons as previously described (Tran et al. 2013). Localization of Nrp2-positive dorsal commissural neurons is unaltered in Nrp2 $2^{f / f}{ }_{\text {F Fox } A 2} 2^{\text {CreERT2+ }}$ mutants. Furthermore, Nrp2 expression in these neurons is comparable between $\mathrm{Nrp}^{\mathrm{f} / \mathrm{f}}{ }_{\text {; Fox } A 2} 2^{\mathrm{CreERT2+}}$ mutants and $\mathrm{Nrp} 2^{\mathrm{f} / \mathrm{f}}$; FOXA2 ${ }^{\text {CreERT2- }}$ controls (Supplemental Fig. 3C,D, area marked by dashed lines). TUNEL (terminal deoxynucleotidyl transferase-mediated deoxyuridine triphosphate nick end labeling) and DAPI labeling reveal no gross changes in apoptosis or cellular density in the spinal cords of $\mathrm{Nrp}^{\mathrm{flf}}{ }_{\text {; FOXA }} 2^{\mathrm{CreERT2+}}+\mathrm{TM}$ mutants compared with no Cre and no TM controls (Supplemental Fig. 4). However, analysis of the ventral commissure in open-book preparation of the $N r p 2^{f / f}$;FoxA2 $2^{\text {CreERT2+ }}+\mathrm{TM}$ mutant spinal cords revealed fewer bundles of crossing axons, consistent with the reduced ventral commissural thickness observed in the transverse sections.

To more broadly extend these findings to other populations of commissural axons, we examined precrossing axon phenotypes in Robo3.1-positive axons and GABAergic commissural neurons that reside in the ventral spinal cord (Phelps et al. 1999). The latter were shown previously to express Nrp2 on their axons (Tran et al. 2013). The normalized fluorescence ratio for both Robo3.1- and GAD65-positive axons is significantly reduced by $\sim 30 \%$ and $>40 \%$, respectively, in $N r p 2^{f / f}$; FOXA2 ${ }^{+/ C r e E R T 2}+\mathrm{TM}$ embryos (Robo3.1, 8.87 \pm 0.51 ; GAD65, 7.66 \pm 1.20 ) compared with untreated controls (Robo3.1, 11.57 \pm 0.57; GAD65, $14.48 \pm 1.06$ ) (Fig. 4A-C; Supplemental Fig. 5A-C). Additionally, the normalized ventral commissure thickness is reduced by $>25 \%$ in Robo3 $11^{+}$and GAD $65^{+}$axons from Nrp2 ${ }^{f / f}{ }_{;}$Fox A2 $2^{+/ C r e E R T 2}$ +TM (Robo3.1, $0.033 \pm 0.0021$; GAD65, $0.040 \pm 0.0032$ ) when compared with $N r p 2^{\text {f/f }} ;$ Fox $A 2^{+/ C r e E R T 2}$ embryos receiving no TM (Robo3.1, $0.047 \pm 0.0015$; GAD65, $0.056 \pm$ 0.0021) (Fig. 4D; Supplemental Fig. 5A,B,E). Furthermore, ventral commissure thickness is reduced by $\sim 60 \%$ in Nrp2 $2^{f / f}{ }_{;}$FoxA2 ${ }^{\text {CreERT2/CreERT2 }}$ +TM embryos (Robo3.1, $0.016 \pm 0.0011$ ) (Fig. 4D). This more severe phenotype is likely attributed to more prompt removal of Nrp2 floor plate expression upon TM induction in mice carrying two copies of Cre, suggesting that floor plate-derived Nrp2 could have an even bigger effect than detected here. Collectively, these findings point to a functional requirement of floor plate-associated Nrp2 in regulating precrossing axon guidance in vivo.

\section{Cell-autonomous function of Nrp2 or PlxnA1 in dorsal commissural neurons is not required for precrossing axon pathfinding}

Nrp2-null embryos show defects in midline crossing and post-crossing axon trajectories (Zou et al. 2000; Tran et al. 2013), but no defects in precrossing axon pathfinding have been reported. Because floor plate-specific deletion of Nrp2 revealed a novel role for Nrp2 in the regulation of precrossing commissural axons, we next examined whether eliminating Nrp2 or PlxnA1 in commissural 


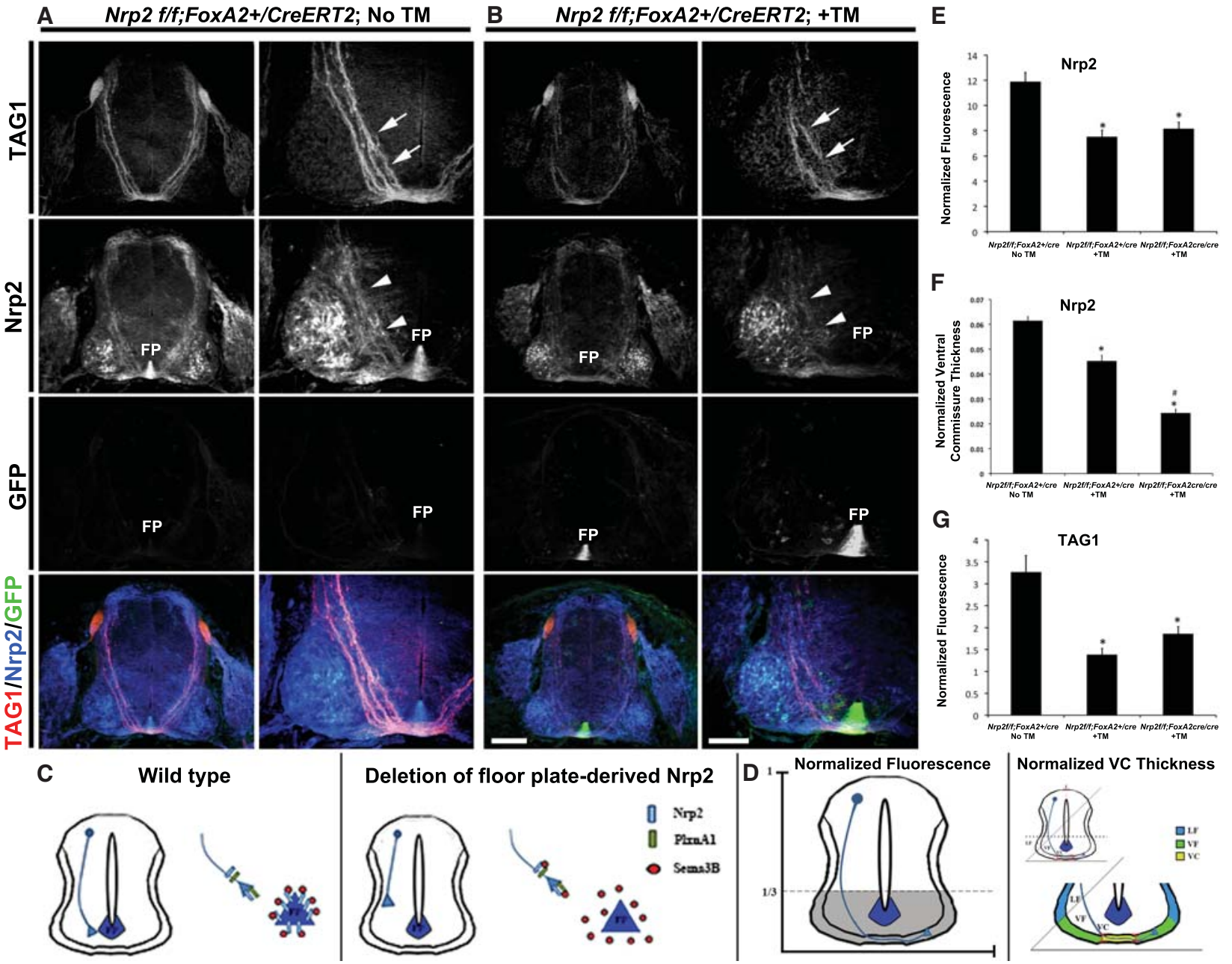

Figure 3. Specific deletion of floor plate-derived Nrp2 revealed precrossing guidance defects in Nrp2-positive commissural axons. $(A, B)$ Representative confocal images of E11.5 spinal cord sections were taken from a Nrp2 $2^{f / f}{ }_{j} F_{O X A} 2^{+/ C r e E R T 2}$ embryo with no TM treatment (No $\mathrm{TM}_{;}$A) or a Nrp2 ${ }^{f / f}$;FoxA2- ${ }^{+}$CreERT2 embryo with TM treatment $(+\mathrm{TM} ; B)$. All transverse sections were processed for immunocytochemistry for TAG1 (red), Nrp2 (blue), and GFP (green). Bars: in left column of $B$ (for $A, B$ ) (low magnification), $160 \mu \mathrm{m}$; in right column of $B$ (high magnification), $80 \mu \mathrm{m}$. White arrows and white arrowheads point to TAG1 ${ }^{+}$and $\mathrm{Nrp}^{+}$axons, respectively. (FP) Floor plate. Nrp2 $2^{f / f}$; FOXA2 ${ }^{\text {CreERT2/CreERT2 }}$ +TM embryos were analyzed, and images are shown in Supplemental Figure 3. (C) Schematic diagrams of E11.5 spinal cords illustrating many fewer dorsal Nrp2 ${ }^{+}$precrossing axons failing to reach the most ventral one-third of the cord and midline when Nrp2 expression is specifically deleted from the floor plate. $(D)$ Schematic diagrams illustrating the parameters for the measurements of the normalized immunofluorescence (left side) and the normalized ventral commissure thickness (right side) (see the Materials and Methods). (E) Quantifications of Nrp2-normalized fluorescence in $N r p 2^{f / f} ;$ Fox $A 2^{+/ C r e E R T 2}$ No TM, Nrp2 ${ }^{f / f}$; FoxA2 $2^{+/ C r e E R T 2}+\mathrm{TM}$, and Nrp2 $2^{f / f}$; FOXA2 ${ }^{\text {CreERT2/CreERT2 }}+$ TM embryos. Data are means \pm SEM from five to six sections per embryo, where $n=5$ embryos per genotype. ANOVA followed by post-hoc Tukey test, $\left({ }^{*}\right) P<0.05$ compared with $N r p 2^{f / f}{ }_{;}$FOX $A 2^{+/ C r e E R T 2}$ No TM. $(F)$ Quantifications of Nrp2-normal-

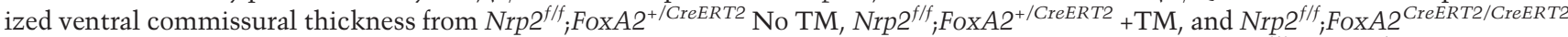
+ TM embryos. Data are means \pm SEM from five to eight sections per embryo, where $n=8$ embryos for Nrp2 $2^{f / f} ;$ FOxA $2^{+/ C r e E R T 2}$ No TM; $\mathrm{Nrp2} 2^{f / f} ;$ FoxA2 $2^{+/ C r e E R T 2}+\mathrm{TM} ; n=7$ embryos for Nrp2 $2^{f / f}$;FoxA2 $2^{\text {CreERT2/CreERT2 }}+\mathrm{TM}$. ANOVA followed by post-hoc Tukey test, $\left({ }^{*}\right) P<$

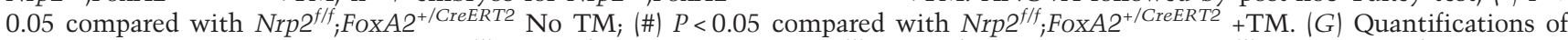

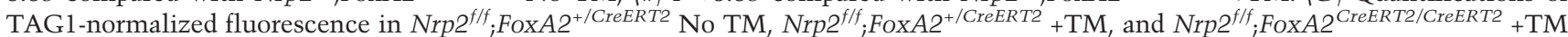
embryos. Data are means \pm SEM from five to six sections per embryo, where $n=5$ embryos for $N r p 2^{f / f} ;$ FOxA $2^{+/ C r e E R T 2} \mathrm{No}$ TM; $n=3$ em-

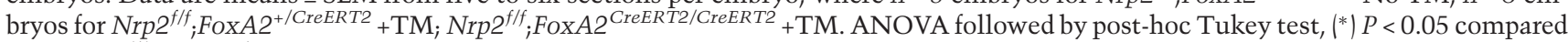
with Nrp2 $2^{\text {flf }}$ FOXA2 $2^{+/ C r e E R T 2}$ No TM.

neurons would reveal a cell-autonomous role for this signaling pathway in precrossing commissural axon guidance. The majority of Nrp2-positive dorsal spinal commissural neurons arises from Atoh1/Math1 progenitors
(Helms and Johnson 1998; Tran et al. 2013). Therefore, we crossed the Nrp2 $2^{f / f}$ (Walz et al. 2002) and PlxnA1 f/f (Yoshida et al. 2006) conditional knockout mice with a line expressing Cre driven by the Math1 promoter (Matei 

et al. 2005). As with the Nrp2 $2^{f / f}$ line, PlxnA1 f/f mice express GFP in cells following Cre-mediated recombination of the conditional allele. We first examined expression of

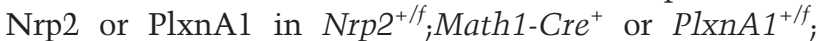
Math1-Cre $e^{+}$E11.5 embryos, respectively, and found that neither protein expression profile was altered (data not shown). Next, we found that E11.5 Nrp2 ${ }^{+/ f} ; M^{M a t h 1-C r e}{ }^{+}$ embryos express Nrp2 and that $\mathrm{PlxnA}^{+/ f}{ }_{\text {iMath1-Cre }}{ }^{+}$ embryos express PlxnAl on their commissural axons at levels comparable with that of wild-type controls (Figs. 1A, 5A; Supplemental Fig. 6), but commissural axon staining for Nrp2 and PlxnA1 is specifically lost in $N r p 2^{f / f}$; Math1:Cre ${ }^{+}$and PlxnA1 ${ }^{f / f}$;Math1-Cre ${ }^{+}$animals, respectively. As above, Robo3.1 was quantified and normalized between animal groups to examine mutants for precrossing deficits. Nrp2 ${ }^{f / f}$; Math1-Cre ${ }^{+}$(Robo3.1, $7.97 \pm 0.69$ ) and PlxnA1 ${ }^{f / f}{ }_{;}$Math1-Cre ${ }^{+}$(Robo3.1, 6.47 \pm 0.40 ) embryos have similar axonal trajectories toward the midline when compared with control littermates $\left(\mathrm{Nrp} 2^{+/ f}\right.$; Math1-Cre ${ }^{+}$[Robo3.1, 7.48 \pm 0.42], PlxnA1 ${ }^{+/ f}{ }^{\text {; Math1- }}$ $\mathrm{Cre}^{+}$[Robo3.1, $\left.5.65 \pm 0.32\right]$, and PlxnA1 ${ }^{+/+}{ }_{\text {; Math1-Cre }}^{+}$ [5.48 \pm 0.39$]$ ) (Fig. 5; Supplemental Fig. 6A-D). However, consistent with the post-crossing phenotype observed in E11.5 Nrp2 $2^{-/-}$embryos (Tran et al. 2013), the thickness of ventral commissure and future ventral funiculus, but not the lateral funiculus, is significantly decreased in PlxnA1 ${ }^{+/ f}$; Math1-Cre $e^{+}$(ventral commissure $=0.05392 \pm$ 0.0045 ; ventral funiculus $=0.0724 \pm 0.0033$; lateral funiculus $=0.0248 \pm 0.0019$ ) and PlxnA1 fif ${ }^{\text {Math1-Cre }}{ }^{+}$(ventral commissure $=0.0626 \pm 0.0018$; ventral funiculus $=$ $0.0693 \pm 0.0013$; lateral funiculus $=0.0206 \pm 0.0009)$ spinal cords compared with $\mathrm{PlXnA1^{+/+ }}$;Math1-Cre ${ }^{+}$controls (ventral commissure $=0.0793 \pm 0.0027$; ventral funiculus $=0.0886 \pm 0.0031$; lateral funiculus $=0.0315 \pm 0.0017$ ) (Supplemental Fig. 6E). Similar to the conditional PlxnA1 knockouts, we found no gross precrossing axon guidance defects in the global PlxnA1-1- knockouts compared with wild-type controls (Supplemental Fig. 7). Collectively, these results demonstrate that Sema3-Nrp2/PlxnA1 signaling is dispensable for precrossing, but required for post-crossing, commissural axon pathfinding (Fig. 5K; Supplemental Figs. 6F, 7).

Precrossing axon defects in embryos deficient in floor plate-associated Nrp2 are rescued by inhibition of PlxnA1 signaling in vivo

Our in vivo and in vitro results suggest that floor plateassociated Nrp2 acts as a molecular sink to sequester secreted Sema3B, thereby preventing premature repulsion of precrossing axons. We reasoned that if the precrossing defects observed in $\mathrm{Nrp}^{\mathrm{f} / \mathrm{f}}{ }_{\text {; FOX }} \mathrm{A} 2^{+/ C r e E R T 2}$ embryos were mediated by Sema3B that has been liberated by loss of Nrp2 in the floor plate, then disrupting Nrp2/PlxnA1 signaling in precrossing commissural axons should restore normal precrossing pathfinding. To test this hypothesis in vivo, Nrp2 $2^{f / f}$;Fox $A 2^{+/ C r e E R T 2}$ animals were crossed to $P \operatorname{lx} n A 1^{-/-}$knockout mice to generate $N r p 2^{f / f}$;FOXA2 $2^{\text {CreERT2/CreERT2 }}$ PlxnA1 ${ }^{-/-}+\mathrm{TM}$ (no floor plate Nrp2 and axonal Plxn), Nrp2 ${ }^{+/ f}{ }_{;} F_{O x A} 2^{+/+}$;
PlxnA1 ${ }^{+/-}+$TM (with floor plate Nrp2 and axonal PlxnA1), and Nrp2 ${ }^{f / f}$;FoxA2 ${ }^{+/ C r e E R T 2}{ }_{\text {; PlxnA1 }}{ }^{+/+}+\mathrm{TM}$ (no floor plate Nrp2 but with axonal PlxnA1). As expected, E11.5 Nrp2 ${ }^{+/ f}{ }_{j}$ Fox $A 2^{+/+}{ }_{i} P \operatorname{PnnA1} 1^{+/-}+\mathrm{TM}$ control embryos display no precrossing guidance errors (in $\mathrm{Nrp}^{+}$and Robo3. $1^{+}$axons) (Fig. 6A). Similar to Nrp2 $2^{f / f}$;

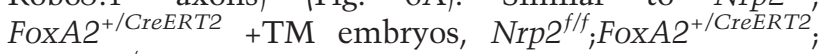
$P l \times n A 1^{+/+}+$TM embryos showed precrossing defects in both Nrp2- and Robo3.1-positive axons when compared with $\mathrm{Nrp2}^{+/ f}{ }_{\text {; Fox }} \mathrm{A2}^{+/+}{ }_{\text {; PlXnA }} 1^{+/-}+\mathrm{TM}$ controls (Fig. 6B). These precrossing guidance errors are rescued in $N r p 2^{f / f}$; FoxA2 ${ }^{\text {CreERT2/CreERT2 }}$; PlXnA1 $1^{-/-}$+TM mutant spinal cords (Fig. 6C). The normalized fluorescence intensity of Robo3.1-positive precrossing axons from $N r p 2^{f / f}$; FoxA2 $2^{+/ C r e E R T 2}$; PlxnA1 ${ }^{+/+}+\mathrm{TM}(3.30 \pm 0.34)$ is significantly decreased by $30 \%$ compared with $\mathrm{Nrp2}^{+/ f}$; Fox $\mathrm{A2}^{+/+}{ }_{;} P \operatorname{Pl} n A 1^{+/-}$+TM controls $(4.78 \pm 0.28)$ but recovered in Nrp2 ${ }^{f / f}{ }_{;}$FoxA $2^{\text {CreERT2/CreERT2 }}{ }^{\text {PlXnA } A 1^{-/-}}$ + TM embryos $(5.73 \pm 0.44)$ (Fig. 6D). Finally, we also observed that the ventral commissure thickness for Robo3.1-positive axons crossing the midline was significantly decreased by $20 \%$ in $\mathrm{Nrp}^{\mathrm{flf}}{ }_{\text {; FOX A }}{ }^{+/ C r e E R T 2}$; $P \operatorname{PnA} 1^{+/+}+\mathrm{TM}(0.0511 \pm 0.001)$ and $29 \%$ in $N r p 2^{f / f}$; FOXA2 ${ }^{\text {CreERT2/CreERT2 }}$; PlxnA1 $1^{-/-}+$TM $\quad(0.0455 \pm 0.002)$ when compared with $\mathrm{Nrp}^{+/ f}$; FoxA2 ${ }^{+/+}{ }_{\text {i }} \mathrm{PlxnA1^{+/- }}+\mathrm{TM}$ $(0.0640 \pm 0.002)$ embryos (Fig. 6E). Taken together, our findings suggest that the Nrp2/PlxnA1 signaling in precrossing axons is attenuated by floor plate-derived Nrp2, but Nrp2/PlxnA1 signaling in response to Sema3B repulsion is required for crossing axons to exit the midline and post-crossing pathfinding (Fig. 6F).

\section{Discussion}

We describe here a novel mechanism for dynamically regulating the responsiveness of precrossing and postcrossing commissural axons to secreted semaphorins derived from the floor plate. First, we demonstrated that dorsal commissural neurons indeed express both Nrp2 and PlxnA1 receptors on both their pre- and post-crossing segments and that these precrossing axons are responsive to Sema3B repulsion in vitro. In addition, we uncovered a previously unappreciated source of Nrp2 expressed by floor plate cells as early as E9.5, before any precrossing axons have reached the ventral commissure in the developing spinal cord. This floor plate-derived Nrp2 is temporally regulated, and its expression levels dramatically increase and peak around E11.5, at the developmental stage when the majority of dorsal commissural axons are crossing the midline. Later in development, Nrp2 is down-regulated (E12.5-E13.5), allowing Sema3B to exert its repulsive effects on post-crossing axons (Fig. 7). Using in vivo mouse genetics, we further demonstrated that this non-cell-autonomous mechanism of floor plate-associated Nrp2 is required for proper precrossing axon guidance. Collectively, our in vivo and in vitro results suggest a novel non-cell-autonomous mechanism by which floor plate-associated Nrp2 serves as a molecular sink to dampen the repulsive effects of 


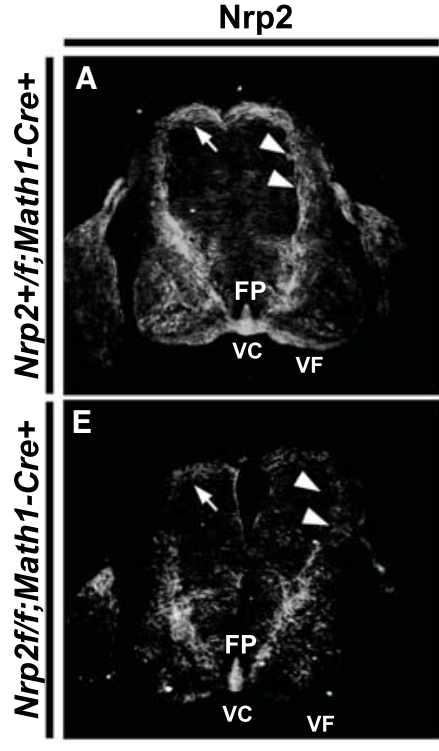

I

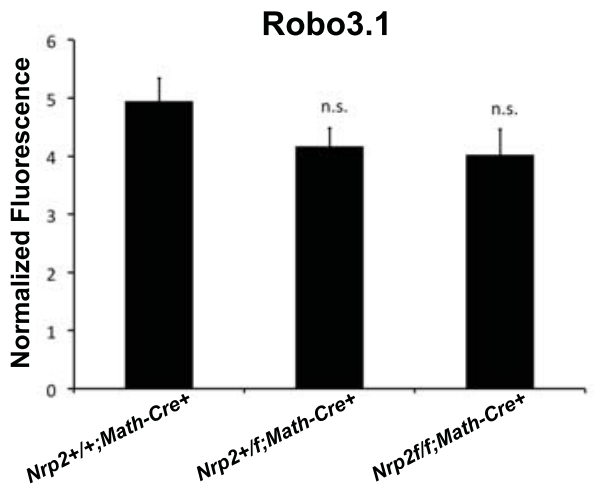

Robo3.1
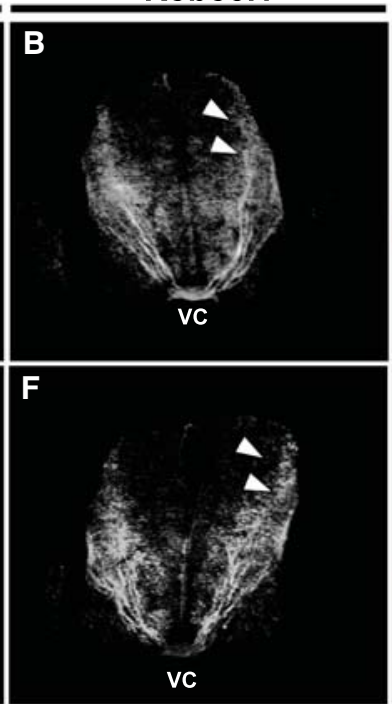

J
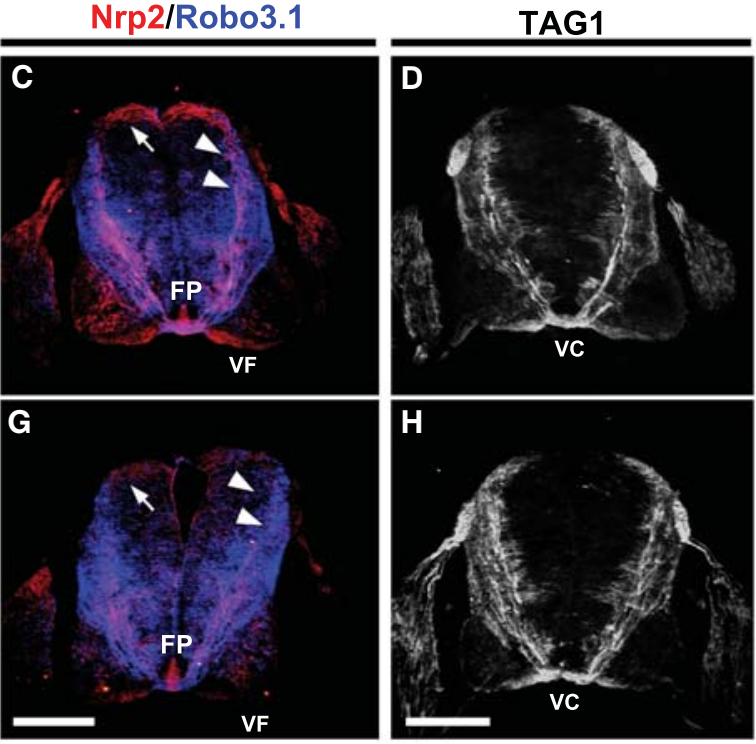

Robo3.1

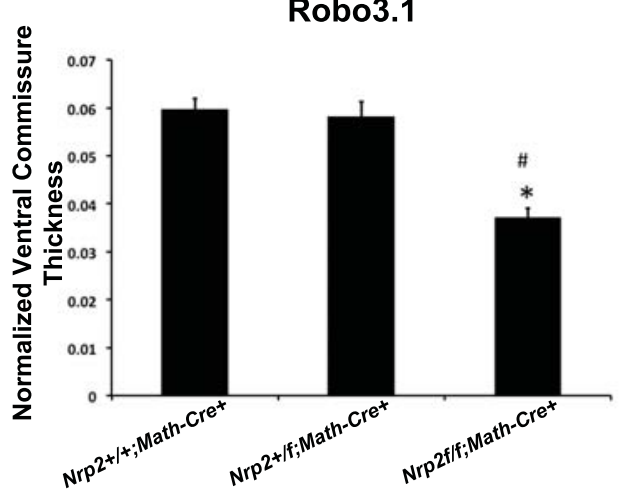

Deletion of Nrp2 in dorsal commissural neurons

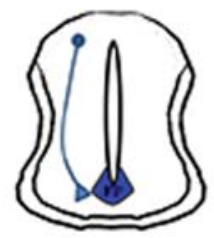

(1) Nip2

(1) Pirail

- $\operatorname{sen} 3 B$

Figure 5. Specific deletion of Nrp2 from dorsal commissural neurons in $\mathrm{Nrp}^{\mathrm{flf}} ; \mathrm{Math1}$-Cre ${ }^{+}$spinal cords shows normal precrossing axon

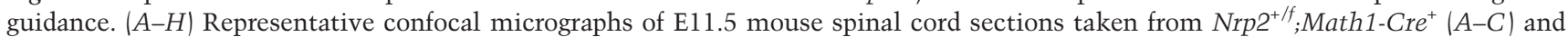
$\mathrm{Nrp}^{f / f}$;Math1-Cre ${ }^{+}(E-H)$ littermate embryos. The same transverse section was processed for immunohistochemistry against Nrp2 (red) and Robo3.1 (blue) in $A-C$ and $E-G$. Bar in $G$ (for $A-C, E-G), 250 \mu \mathrm{m}$. $(D, H)$ Representative confocal micrographs of E11.5 mouse

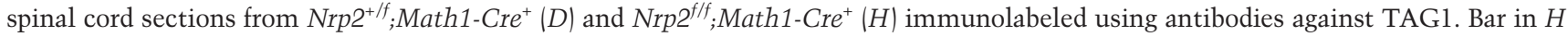
(for $D, H), 250 \mu \mathrm{m}$. White arrows and white arrowheads point to $\mathrm{Nrp}^{+}$cell body location and axons, respectively, in $A, C, E$, and $G$. White arrowheads in $B$ and $F$ point to corresponding Robo3. $1^{+}$axons relative to Nrp2 ${ }^{+}$axons. (FP) Floor plate; (VC) ventral commissure; (VF) ventral funiculus. (I) Quantifications of Robo3.1-normalized fluorescence in precrossing axons showed no significant difference between $\mathrm{Nrp}^{+/+}{ }_{;}$Math1-Cre ${ }^{+}, \mathrm{Nrp}^{+/ f}{ }_{;} \mathrm{Math1} 1-\mathrm{Cre}^{+}$and $\mathrm{Nrp}^{\mathrm{flf}}{ }_{;} \mathrm{Math} 1-\mathrm{Cre}^{+}$littermates. Data are means \pm SEM from five sections measured per embryo, where $n=5$ embryos per genotype. ANOVA, (n.s.) not significant. (J) Quantifications of normalized ventral commissure thickness in Robo3.1-positive precrossing axons in $\mathrm{Nrp2}^{+/+}{ }_{;} \mathrm{Math}_{1-\mathrm{Cre}^{+}}$and $\mathrm{Nrp}^{+/ /}{ }_{;}$Math1-Cre ${ }^{+}$littermates show no significant difference. A significant decrease in ventral commissure thickness was observed in $\mathrm{Nrp}^{\mathrm{ffl}}{ }_{;}{ }_{\text {Math1-Cre }}{ }^{+}$compared with littermate controls. Data are means \pm SEM from five to eight sections measured per embryo, where $n=4 \mathrm{Nrp}^{+/ f}{ }_{;}$Math1-Cre ${ }^{+}$and $n=3 \mathrm{Nrp} 2^{f / f}{ }_{;}$Math1-Cre $e^{+}$. ANOVA, post-hoc Tukey, $\left({ }^{*}\right) P<0.05$ compared with $\mathrm{Nrp}^{+/+}{ }_{;}$Math1-Cre ${ }^{+} ;(\#) P<0.05$ compared with $\mathrm{Nrp2}^{+/ f}{ }_{;}$Math1-Cre ${ }^{+}$. $(\mathrm{K})$ Schematic diagram illustrating wild-type (left) and axonal deletion of Nrp2 (right) genotypes and phenotypes observed in E11.5 spinal cords. Analysis of specific deletion of PlxnA1 in dorsal commissural neurons and PlxnA1 global knockout mutants are shown in Supplemental Figures 6 and 7, respectively. 
A Nrp2+/f; FoXA2+/+;
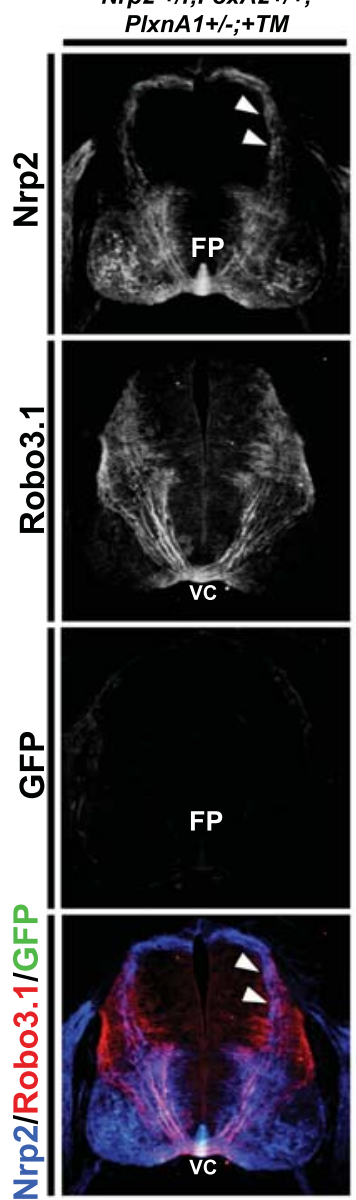

C Nrp2 f/f;FoxA2Cre/Cre; D
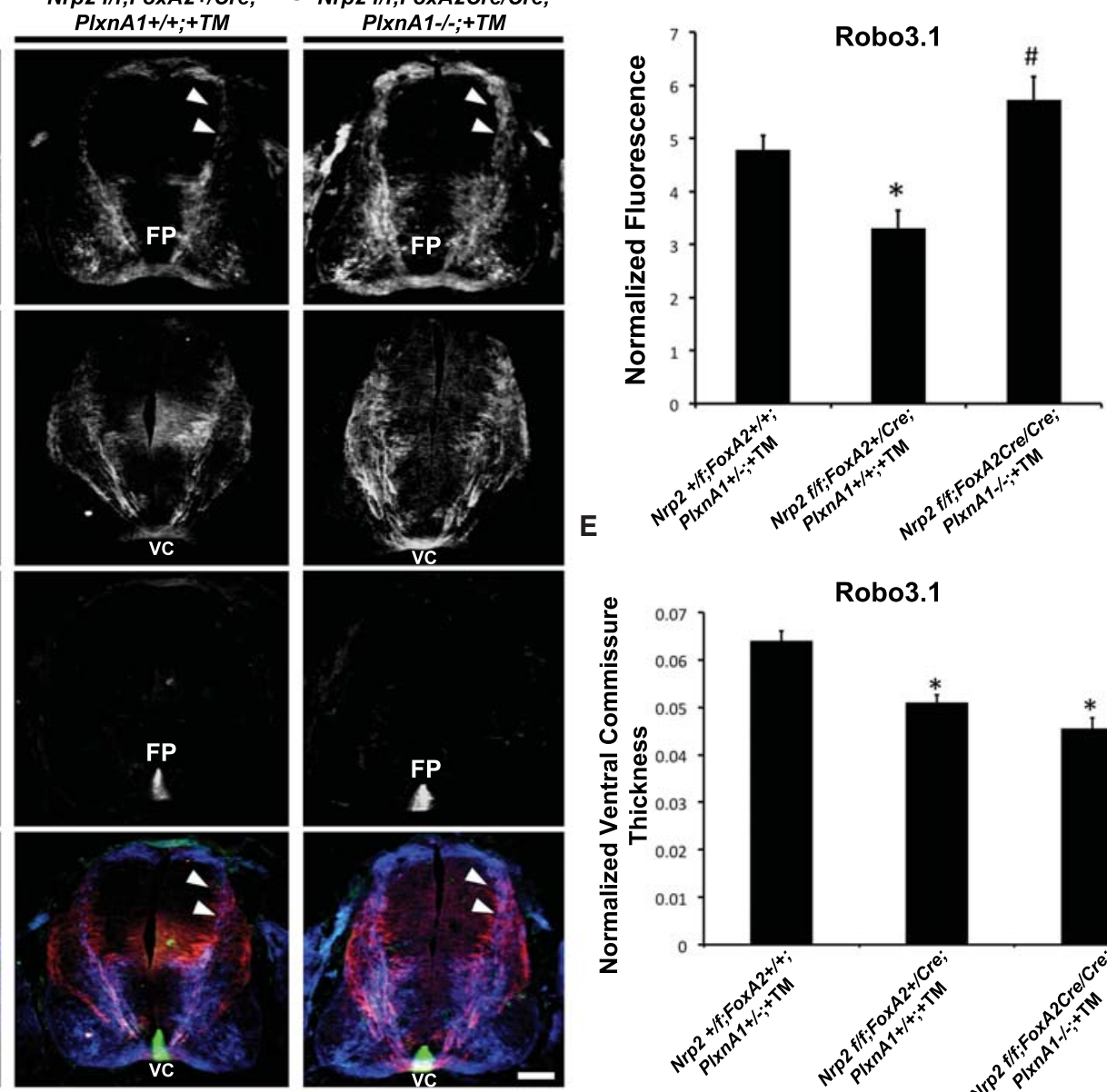

$\mathbf{F}$

Schematic diagram of in vivo mouse genetics and their corresponding phenotypes

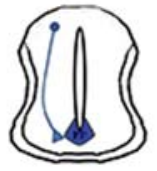

Nrp2+/f;FoxA2+/+;PIXnA1+/-

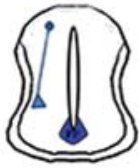

Nrp2f/f;FoxA2+/Cre;PIXnA1+/+

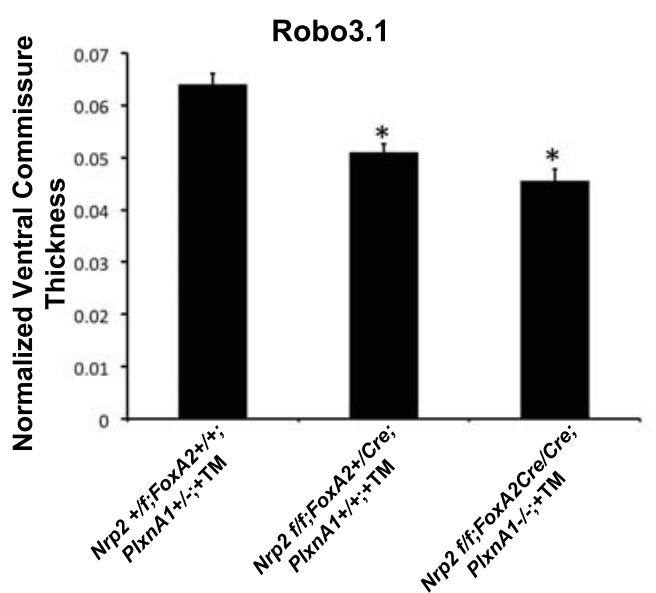

0 Nrp2

I] PIxnA1

- Sema3B

Figure 6. Precrossing axon guidance defects from floor plate-derived Nrp2-deficient animals are rescued by inhibition of PlxnA1 signaling in vivo. $(A-C)$ Representative confocal images of E11.5 spinal cord sections taken from littermates treated with TM (+TM)

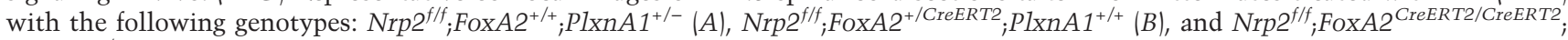
PlxnA1 ${ }^{-/-}(C)$. All transverse sections were processed for immunocytochemistry for Nrp2 (blue), Robo3.1 (red), and GFP (green). White arrowheads illustrate Nrp2-positive dorsal commissural axons projecting toward the floor plate (FP) and ventral commissure (VC)

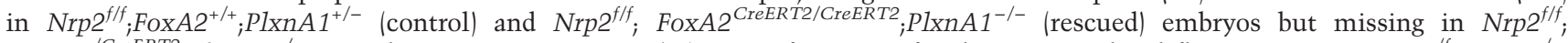
FOXA2 ${ }^{+/ C r e E R T 2}{ }_{\text {} P l X n A 1}{ }^{+/+}$animals. Bar, $A-C, 125 \mu \mathrm{m}$. $(D)$ Quantifications of Robo3.1-normalized fluorescence in $N r p 2^{+/ f}{ }_{;} F_{O X A 2}{ }^{+/+}$;

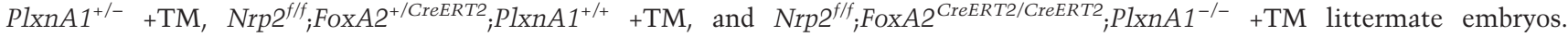
Data are means \pm SEM from five to eight sections per embryo, where $n=3$ embryos per genotype combination analyzed. ANOVA followed

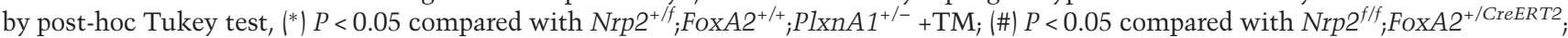

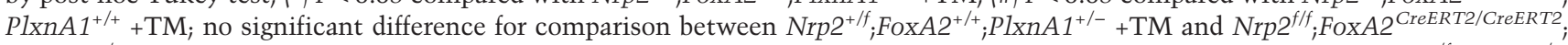

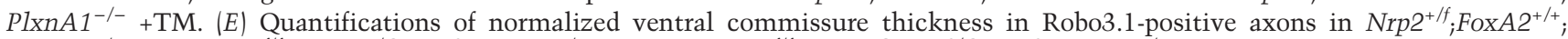

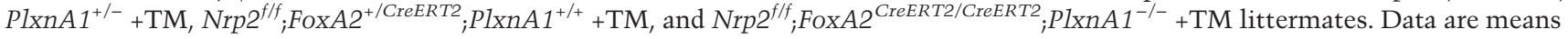
\pm SEM from five to eight sections per embryo, where $n=3$ embryos per genotype combination analyzed. ANOVA followed by post-hoc Tukey test, $\left(^{*}\right) P<0.05$ compared with $\mathrm{Nrp}^{+/ f}{ }_{;} \mathrm{FOXA}_{\mathrm{A}} 2^{+/+}{ }_{;} P \operatorname{lxnA1} 1^{+/-}+\mathrm{TM}$. $(F)$ Schematic diagram of in vivo mouse genetics and the corresponding phenotypes observed. 


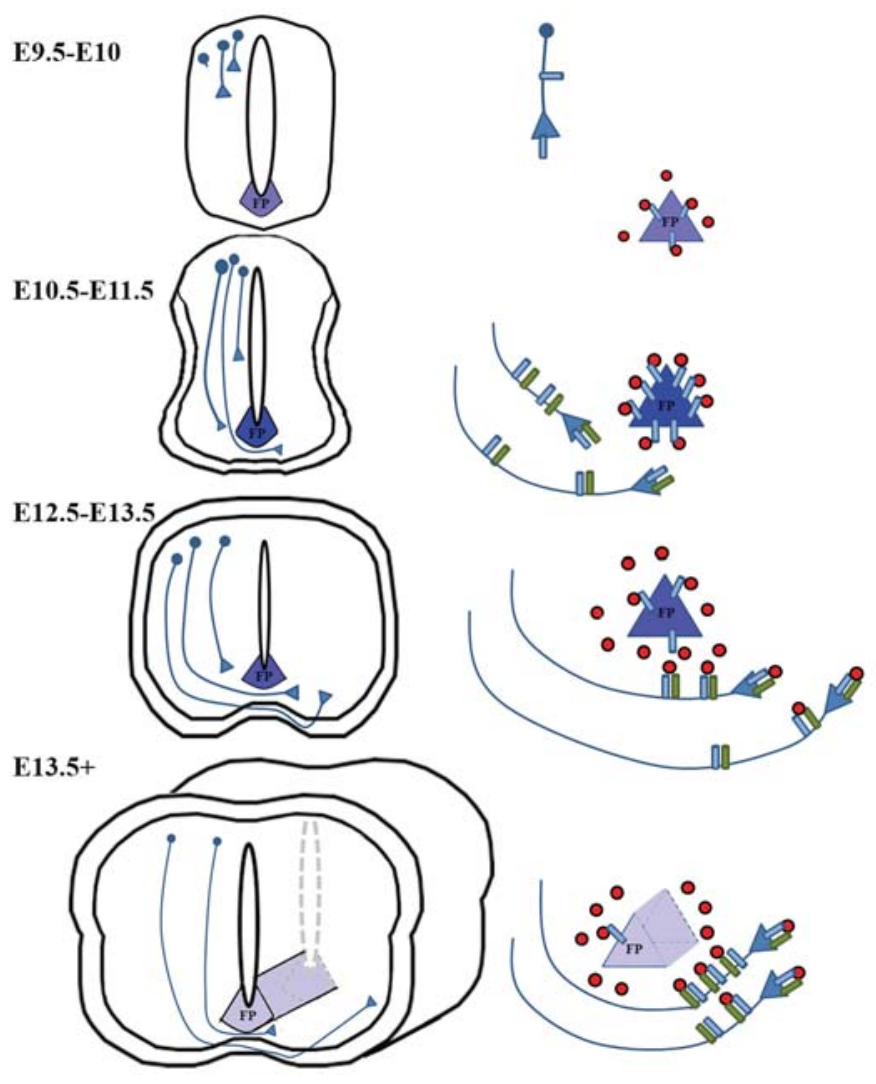

Nip2 Figure 7. Model of Nrp2 function in mediating axoNrp2 nal guidance of dorsal commissural neurons in the dePIxnA1 veloping spinal cord. A schematic diagram based on O Sema3B the in vivo and in vitro results illustrates Nrp2 expression observed as early as E9.5-E10.5 in the floor plate, when only few commissural neurons have begun to extend their axons. As precrossing axons approach the midline, from E10.5 to E11.5, floor plate expression of Nrp2 reaches its peak, resulting in the sequestering of Sema3B and thus allowing Nrp2/PlxnA1positive axons to cross. When most of the commissural axons have crossed the midline, at E12.5-E13.5, floor plate Nrp2 is dramatically down-regulated, releasing Sema3B and promoting the exit of Nrp2/ PlxnA1-positive axons away from the midline. From E13.5 on, the localization of axonal Nrp2 to the post-crossing segments of commissural axons coupled with the near absence of floor plate-derived Nrp2 provides guidance along the longitudinal axis toward brain targets while preventing the recrossing of the midline.
Sema3B on precrossing axons expressing Nrp2 and PlxnA1 (Fig. 7).

\section{Silencing semaphorin signaling on precrossing commissural axons}

Commissural axons achieve midline crossing by changing their response to the floor plate from attraction to repulsion (Stein and Tessier-Lavigne 2001; Chédotal 2010; Derijck et al. 2010; Yam et al. 2012), but it is critical that premature repulsion is avoided to allow precrossing commissural axons to first reach the floor plate. Silencing of repulsion in precrossing axons can be achieved by both intrinsic and extrinsic mechanisms. In the mammalian spinal cord, Robo3 provides perhaps the prototypical example of how this is regulated at the molecular level through noncanonical roles for guidance receptors. Robo3 silences premature Robo1/2-mediated Slit repulsion in precrossing axons but is down-regulated after midline crossing to permit Slit-mediated repulsion of post-crossing axons (Sabatier et al. 2004; Chen et al. 2008; Jaworski et al. 2010). In Drosophila, a similar but distinct in cis mechanism ensures proper commissural formation. The endosomal sorting receptor Commissureless (Comm) antagonizes Slit-mediated repulsion of precrossing axons by down-regulating Robol expression in precrossing axons (Kidd et al. 1998b; Keleman et al. 2002, 2005), thereby preventing premature Slit repulsion.
Low levels of Robol receptors still escape Comm-dependent sorting (Kidd et al. 1998a), raising the question of how their functions are suppressed in the precrossing commissural axons. A recent study revealed that Robo2 is expressed by midline glial cells where they interact in trans with Robol receptors on precrossing axons and prevents canonical Slit-Robol repulsion (Evans et al. 2015). Indeed, overexpression of Robo 2 in midline glia cells suppresses Comm mutant phenotypes in Drosophila.

This Robo receptor model cannot be applied to the mammalian spinal cord because Robo receptors (Robol/ $2 / 3$ ) are not expressed in the mouse floor plate. Instead, we found that the Nrp2 receptor, which signals semaphorin repulsion in commissural axons, is highly expressed in the floor plate. Nrp2 receptors in the floor plate act as a sink for semaphorin ligands to attenuate commissural axon repulsion during midline attraction. Therefore, our Nrp2 model would serve as the vertebrate counterpart for the trans-acting mechanism of the Drosophila Robo2 model.

These two non-cell-autonomous functions of guidance receptors not only point to the floor plate as a source for attractive and repulsive guidance cues but show that the floor plate also regulates the availability of these cues. A similar mechanism was recently identified by another study, which showed that glycosylated dystroglycan could directly bind Slit and anchor its activity at the midline (Wright et al. 2012). 
Semaphorin signaling on pre- and post-crossing commissural axons

Our detailed analysis of the spatial and temporal expression profile of Nrp2 in the developing mouse spinal cord revealed a previously unrecognized mechanism for the regulation of commissural axon midline guidance by which local semaphorin signaling is silenced or attenuated at the time when precrossing commissural axons project toward the ventral midline. Nrp2 transcripts were previously shown to be highly expressed at the floor plate (Chen et al. 1997), and, in the present study, we confirmed this observation of cell type-specific Nrp2 expression by coimmunolabeling Nrp2 and the floor plate-specific murine SC1-related protein Ben. Nrp2 binds with high affinity to Sema3F (Chen et al. 1997, 1998; Giger et al. 1998), and phenotypic analyses of mouse mutants have demonstrated in vivo functional roles for Sema3F-Nrp2/PlxnA3 signaling (Huber et al. 2005; Yaron et al. 2005; Tran et al. 2009). However, much less is known about the receptor complex that mediates Sema3B signaling despite Sema3B being robustly expressed in the rodent spinal cord during commissural axon pathfinding (Zou et al. 2000).

In situ hybridization analysis reveals that PlxnAl is highly expressed in commissural neurons in the developing spinal cord and is therefore the most likely candidate for forming a receptor complex with Nrp2 to convey Sema3B signals (Nawabi et al. 2010). Our analysis is consistent with previous findings that show that PlxnAl is detected on crossing and post-crossing commissural axons (Nawabi et al. 2010). Importantly, however, we also found that PlxnA1 is also prominently expressed on precrossing segments. In some sections and open-book preparations of spinal cords, PlxnAl appears elevated in the ventral commissure, but this increase in fluorescence intensity more likely reflects the convergence of commissural axons within the restricted space of the ventral floor plate than a change in receptor expression. The question of whether PlxnAl levels on pre- and post-crossing axons are modulated is interesting, but, due to their highly fasciculated and bundled nature, especially at the midline region, it is not feasible to accurately quantify PlxnAl levels along the individual precrossing, crossing, or postcrossing segments in vivo. Furthermore, whether the sensitivity to secreted semaphorins changes following midline crossing and whether the dynamics in receptor complex composition also changes after midline crossing remain to be addressed by future experiments.

On the other hand, Sema3F preferentially signals through Nrp2/PlxnA3 receptor complexes, which likely accounts for the response of post-crossing axons to Sema3F. In vivo, Sema3F expression is detected in the floor plate of E12-E13.5 mouse spinal cords (Zou et al. 2000; Cohen et al. 2005), after the majority of commissural axons have crossed the midline, which would be consistent with Sema3F regulating contralateral axonal pathfinding of commissural axons. Supporting this model, Sema3F repels post-crossing axons in vitro (Supplemental Fig. 2B; Zou et al. 2000). However, Sema3F has no effect on dorsal precrossing axons (Fig. 1H; Zou et al. 2000), raising the question of how Sema3F sensitivity is regulated during midline crossing for future analysis.

\section{Dynamic spatial and temporal expression of Nrp2 and PlxnA1 in the mouse spinal cord}

The dynamically regulated floor plate expression profile of Nrp2, coupled with its interactions with secreted semaphorins, places floor plate-derived Nrp2 in the appropriate spatial and temporal setting to regulate semaphorin-mediated signaling during midline crossing. Floor plate-associated Nrp2 is only required to serve as a Sema3B chelator if precrossing axons coexpress Nrp2 and PlxnA1, as we show here. This finding appears to contradict previously published studies using a variety of in vitro assays that were unable to elicit a Sema3B response in precrossing axons (Zou et al. 2000; Nawabi et al. 2010). This may explain the differences in the culture system that was used. Previously, other laboratories have used Sema3B-expressing HEK cell aggregates or floor plate-conditioned medium, which could make it difficult to control for the amount of Sema3B present in the culture system. The floor plate also expresses many other factors that could confound the effect of Sema3B. Therefore, to circumvent these caveats, we used different dosages of recombinant Sema3B required for axonal inhibition with a larger dynamic range. We were able to observe the post-crossing effect as previously reported, which served as a positive control for our precrossing analysis. While we observed a strong effect on the post-crossing outgrowth, the suppression is actually not complete (Supplemental Fig. 2) with either Sema3B or Sema3F, even at high concentrations. This should be expected given the heterogeneous nature of the different commissural populations, suggesting that our cultures were raised in a healthy condition that can recapitulate the endogenous situation. To study the precrossing effect directly, we used isolated E11.5 spinal cord explants restricted to the most dorsal region. The robust and consistent outgrowth from this more defined region gave us a large and sensitive range to test Sema3B's effects. They are indeed slightly less sensitive (32\% reduction) compared with the mixed population of post-crossing commissural axons ( $48 \%$ reduction) at lower concentrations $\mid 0.5$ $\mu \mathrm{g} / \mathrm{mL}$ ); however, they can be fully suppressed at higher concentrations, which is consistent with the fact that they are mostly Nrp2-positive.

A key finding that motivated us to investigate the functional role of floor plate-derived Nrp2 was the discovery that PlxnAl is robustly expressed on precrossing segments of commissural axons. Previous work failed to detect PlxnA1 expression on precrossing commissural axons using a commercial rabbit polyclonal antibody (Abcam) (Nawabi et al. 2010), but, using our protocols for immunohistochemistry, we found that the staining of commissural axons by this antibody was nonspecific, as assessed using PlxnA1-null mice. Therefore, we reexamined the PlxnAl expression profile using another rabbit polyclonal antibody (Yoshida et al. 2006), which

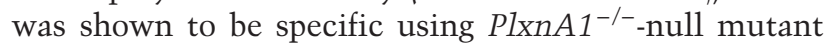
spinal cords. Our results confirm that the anti-PlxnA1 
from Yoshida et al. (2006) was indeed specific and that PlxnA1 is expressed on precrossing axons at different developmental stages that coincide with Nrp2 expression. Furthermore, we used a nonpermeabilized staining protocol to optimize the visualization of Nrp2 and PlxnA1 on the precrossing axon surface, where they can be responsive to extracellular cues (data not shown). Moreover, in support of our expression analyses, we demonstrated that precrossing axons are indeed sensitive to Sema3Bmediated inhibition in a three-dimensional spinal cord explant assay (Xu et al. 2014).

\section{Mechanism of floor plate-associated Nrp2 function at the midline}

We show here that Nrp2 is expressed at high levels in both precrossing and post-crossing commissural axons. However, Nrp2 $2^{-/-}$spinal commissural axons show guidance errors only in the post-crossing portion of their axon trajectories or as their axons exit the midline; we and others observed no precrossing axon pathfinding defects (Zou et al. 2000; Tran et al. 2013; this study). Our results from Nrp2 ${ }^{f / f}{ }_{;}$Math1-Cre ${ }^{+}$conditional embryos in which Nrp2 is specifically removed in dorsal commissural neurons corroborate previous findings. Because Sema3B is highly expressed at the floor plate when commissural axons begin projecting toward the midline and throughout the time they exit the floor plate, precrossing axons may lack the appropriate receptor complex on their growth cone surface to signal Sema3B repulsion. However, we found that both Nrp2 and PlxnA1 are highly expressed in precrossing commissural axons, and our in vitro studies demonstrate that these precrossing axons are responsive to exogenously applied Sema3B. This suggests an additional layer of complexity for regulating Sema3B-mediated signaling in the developing spinal cord. We found that Nrp2 expression at the floor plate plays a significant role in ensuring that precrossing commissural axons properly reach the floor plate. Therefore, it is unlikely that these precrossing guidance defects are due to a cell-autonomous function of Nrp2 in floor plate development, since we did not observe any overall defects in floor plate morphology, and, furthermore, this phenotype is not observed in Nrp2 knockout embryos.

Our results are consistent with a model in which Nrp2 at the floor plate chelates Sema3B during precrossing extension toward the midline (E10-E11.5), thereby preventing activation of Nrp2/PlxnA1 complexes on precrossing axons. In the absence of floor plate Nrp2, Sema3B is free to act on precrossing axons and exert its repulsive effects on precrossing axons as they enter the ventral spinal cord and approach the floor plate, as we observed in Nrp2 $2^{f / f}$;FoxA2-CreERT2 +TM embryos. While there are no Sema3B-specific antibodies at present to directly monitor this process, in this study, we genetically tested this hypothesis. If, as our in vitro assays predict, Nrp2/PlxnA1 complexes on precrossing axons are able to functionally respond to Sema3B and if Sema3B is not sequestered at the floor plate, as in the scenario when Nrp2 is specifically deleted from the floor plate, we expect that removal of the PlxnA1 receptor from commissural neuron axons should rescue the precrossing phenotypes observed in $\mathrm{Nrp}^{f / f}$;FoxA2-CreERT2+TM embryos. Indeed, we observed a significant rescue of this precrossing axon phenotype in the Nrp2 $2^{f / f}$;FoxA2-CreERT2+TM;PlXnA1 ${ }^{-1-}$ mice compared with the littermate controls.

Our model of floor plate-derived Nrp2 function at the midline in a non-cell-autonomous manner with respect to commissural neurons is reminiscent of the regulation of retinal ganglion cell axon midline crossing at the optic chiasm by Sema6D/PlxnA1/NrCAM signaling (Kuwajima et al. 2012). Midline cells of the optic chiasm express PlxnA1, which suppresses the inhibitory effects of Sema6D on retinal ganglion cell axons as they cross the CNS midline. Taken together, our study provides new insight into midline axon guidance by demonstrating in vitro and in vivo non-cell-autonomous regulation of Sema3B-Nrp2/PlxnA1 signaling in spinal commissural neurons by which Nrp2 expressed by the floor plate serves as a molecular sink to attenuate the repulsive effect of Sema3B on precrossing commissural axons. Our findings also shed light on the diverse and complex molecular mechanisms exhibited by the spinal cord floor plate to ensure commissural axon navigation toward and across the midline.

\section{Materials and methods}

\section{Mouse strains}

For timed pregnancies, the morning of the day on which a copulatory plug was detected was designated as E0.5. All genetically modified mice were identified by PCR, and DNA samples were taken from tail tissue biopsies. Genotyping was determined as described for Nrp2 knockout (Giger et al. 2000), Nrp2 conditional (Walz et al. 2002), PlxnAl knockout and PlxnAl conditional (Yoshida et al. 2006), Atoh1/Math1-Cre (Matei et al. 2005), and FoxA2-CreER (Park et al. 2008). The Institutional Animal Care and Use Committee (IACUC) of Rutgers University (\#12-027) and Rockefeller University (\#14704-H) approved the animal use protocol.

\section{TM treatment}

TM (Cayman Chemical, 13258) was dissolved in peanut oil at a concentration of $10 \mathrm{mg}$ per $100 \mu \mathrm{L}$. To induce Cre activity for specific deletion of Nrp2 in GABAergic ventral commissural neurons or floor plate cells, pregnant mice were given oral gavage of $10 \mathrm{mg}$ of TM at E6.5 and/or E8.5 using an animal gauge feeding needle as previously described (Park et al. 2008).

\section{Immunohistochemistry}

Immunohistochemistry was performed as described previously (Phelps et al. 1999; Tran et al. 2013). For details, see the Supplemental Material. The following primary antibodies were used in this study: rabbit anti-hPlxnA1 (1:500; Abcam, Ab23391); rabbit anti-mPlxnA1 (1:1500) (Yoshida et al. 2006), rabbit anti-Nrp2 (1:500; Cell Signaling, D39A5), $4 \mu \mathrm{g} / \mathrm{mL}$ goat anti-Nrp2 (R\&D Systems, AF567), anti-Ben (1:100) (Dillon et al. 2005), rabbit anti-GAD65 (1:100; Cell Signaling, 5843), mouse IgM anti-4D7/ TAG-1 (1:800; Developmental Studies Hybridoma Bank, clone 
4D7), goat anti-TAG1 (1:500; R\&D Systems, AF1714), goat antirobo3.1 (1:500; R\&D Systems, AF3076), and mouse anti-GFP (1:800, A11120, Molecular Probes). The following secondary antibodies were used in this study: Alexa fluor 488 donkey antimouse IgG (1:500; Jackson ImmunoResearch Laboratories, 715545-150), Alexa fluor 488 donkey anti-rabbit IgG (1:800; Jackson ImmunoResearch Laboratories, 711-175-152), Cy5 donkey anti-rabbit IgG (1:500; Jackson ImmunoResearch Laboratories, 711-175-152), Cy5 donkey anti-goat IgG (1:500; Jackson ImmunoResearch Laboratories, 705-175-147), Alexa fluor 488 goat anti-mouse IgM (1:800; Jackson ImmunoResearch Laboratories, 115-546-020), Alexa fluor 546 donkey anti-goat IgG (1:500; Molecular Probes, A-11056), and Alexa fluor 546 goat anti-mouse IgM (1:500; Molecular Probes, A-21045). For consistency, immunohistochemistry was performed on serial sections of the same embryo or carefully age-matched and spinal level-matched sections of different embryos.

\section{TUNEL staining}

Apoptotic cell death was analyzed through the processing of mouse spinal cord sections with a TUNEL assay kit (Promega, G3250) following the manufacturer's instructions. Permeabilization incubation times and reagent concentrations were optimized to ensure tissue adherence to the slides. Sections were incubated for $10 \mathrm{~min}$ with $0.8 \%$ Triton-X plus $20 \mu \mathrm{g} / \mathrm{mL}$ proteinase $\mathrm{K}$ solution at room temperature. For positive controls, an additional incubation step was included in which tissue sections were treated with $10 \mathrm{U} / \mathrm{mL}$ DNase I (Thermo Scientific) solution for $5 \mathrm{~min}$ at room temperature. Note that the signal observed in the floor plate was from the endogenous GFP signal from the $\mathrm{Nrp}_{2}{ }^{f / f}{ }_{\text {; }}$ FOXA2CreERT2 $2^{+}$TM animals, indicating that Nrp2 is specifically deleted from the floor plate cells; no TUNEL signaling was detected in the floor plate. The images were captured using a 10× objective on a Zeiss Cell Observer SD confocal microscope.

\section{Analysis of commissural axonal projections and quantifications}

Immunofluorescently labeled cervical level spinal cord sections (20- $\mu \mathrm{m}$ thickness per section) were analyzed and photodocumented using a confocal microscope (Zeiss AxioExaminer Z1). All compiled $Z$-stack images were exported as TIF files to ImageJ (National Institutes of Health) for measurement of the normalized fluorescence ratio. For each section, the corrected total fluorescence $[\mathrm{CTF}=$ integrated density $-($ area selected $) \times($ mean fluorescence of background)] was measured for the ventral-most one-third of the spinal cord (from the most dorsal motor neuron pool to the edge of the marginal zone). Background fluorescence was taken from the ventricular zone of each section measured. The CTF was then normalized to the total area of each section measured. The values of the thickness of the ventral commissure, ventral funiculus, and lateral funiculus were normalized to the distance between roof plate and floor plate for each section, similar to as described previously (Jaworski et al. 2010). For both normalized fluorescence and thickness values, five to eight spinal sections were analyzed per embryo. All measured values were plotted as means \pm SEM from $n=3-5$ embryos per genotype. Statistical analyses were performed with OriginPro 9.1 software. Statistical significance between two samples was determined using two-tailed Student's $t$-test for independent samples. Statistical analyses among multiple groups were determined by using ANOVA followed by post-hoc analysis with Tukey's pairwise comparison test. The criterion for statistical significance was set at $P<0.05$.

\section{Three-dimensional spinal cord explant cultures and neurite quantification}

Spinal cord explants were dissected and cultured as previously described (Xu et al. 2014). Spinal cord explants containing only precrossing axons were microdissected from dorsal spinal cord tissues (see Fig. 1E; Supplemental Fig. 2A), while explants containing post-crossing axons were taken from the entire hemisegment of the spinal cord, including the ventral floor plate tissue (see Supplemental Fig. 2A). Recombinant Netrin-1, Sema3A, Sema3B, and Sema3F proteins were purchased from R\&D Systems. Netrin-1 was used at $250 \mathrm{ng} / \mathrm{mL}$ for all conditions, with semaphorin ligands added at each indicated concentration. Explant outgrowth was measured and quantified as previously described (Xu et al. 2014), and compiled data represent the mean of all cultures analyzed and normalized to the untreated controls, expressed as percentages \pm SEM. Statistical analyses among multiple groups were determined by using ANOVA followed by posthoc Tukey's pairwise comparison test; $n=4$ independent cultures per condition analyzed. The criterion for statistical significance was set at $P<0.05$.

\section{Acknowledgments}

We thank Alex Kolodkin (Johns Hopkins University School of Medicine) for critically reading the manuscript, and Wilma Friedman (Rutgers University) for helpful discussion. We acknowledge Beril Ozden for her excellent technical support in mouse genotyping. Funding from the New Jersey Commission on Spinal Cord Research to T.S.T., and the Minority Biomedical Research Support Program to E.M. supported this work. T.S.T. conceived and designed the project and wrote the manuscript. B.H.-E. performed the majority of the in vivo knockout mouse experiments; Z.W. performed the in vitro spinal cord explant cultures and the open-book preparation experiments and analyzed the data; E.M. assisted with some of the in vivo knockout mouse and openbook experiments, analyzed the data, and made all of the summary diagrams, the table, and the figures. O.O., Z.K., P.F.M., Y. Y., M.T.-L., and T.S.T. analyzed the data and critically discussed and interpreted the results.

\section{References}

Chédotal A. 2010. Further tales of the midline. Curr Opin Neurobiol 21: 68-75.

Chen H, Chédotal A, He Z, Goodman C, Tessier-Lavigne M. 1997. Neuropilin-2, a novel member of the neuropilin family, is a high affinity receptor for the semaphorins Sema E and Sema IV but not Sema III. Neuron 19: 547-559.

Chen H, He Z, Bagri A, Tessier-Lavigne M. 1998. Semaphorinneuropilin interactions underlying sympathetic axon responses to class III semaphorins. Neuron 21: 1283-1290.

Chen H, Bagri A, Zupicich J, Zou Y, Stoeckli E, Pleasure SJ, Lowenstein DH, Skarnes WC, Chédotal, Tessier-Lavigne M. 2000. Neuropilin-2 regulates the development of selective cranial and sensory nerves and hippocampal mossy fiber projections. Neuron 25: 43-56.

Chen Z, Gore BB, Long H, Ma L, Tessier-Lavigne M. 2008. Alternative splicing of the Robo3 axon guidance receptor governs the midline switch from attraction to repulsion. Neuron 58: 325-332.

Claudepierre T, Koncina E, Pfrieger F, Bagnard D, Aunis D, Reber M. 2008. Implication of neuropilin 2/semaphorin $3 \mathrm{~F}$ in retinocollicular map formation. Dev Dyn 237: 3394-3403. 
Cloutier J, Giger R, Koentges G, Dulac C, Kolodkin A, Ginty D. 2002. Neuropilin-2 mediates axonal fasciculation, zonal segregation, but not axonal convergence, of primary accessory olfactory neurons. Neuron 33: 877-892.

Cohen S, Funkelstein L, Livet J, Rougon G, Henderson CE, Castellani V, Mann F. 2005. A semaphorin code defines subpopulations of spinal motor neurons during mouse development. Eur J Neurosci 21: 1767-1776.

Demyanenko G, Riday T, Tran T, Dalal J, Darnell E, Brennaman LH, Sakurai T, Grumet M, Philpot BD, Maness PF. 2011. NrCAM deletion causes topographic mistargeting of thalamocortical axons to the visual cortex and disrupts visual acuity. $J$ Neurosci 31: 1545-1558.

Derijck AA, Van Erp S, Pasterkamp RJ. 2010. Semaphorin signaling: molecular switches at the midline. Trends Cell Biol 20: 568-576.

Dillon A, Fujita S, Matise M, Jarjour A, Kennedy T, Kollmus H, Arnold HH, Weiner JA, Sanes JR, Kapriellan Z. 2005. Molecular control of spinal accessory motor neuron/axon development in the mouse spinal cord. J Neurosci 25: 10119-10130.

Evans TA, Santiago C, Arbeille E, Bashaw GJ. 2015. Robo2 acts in trans to inhibit Slit-Robo1 repulsion in pre-crossing commissural axons. Elife 4: e08407.

Giger R, Urquhart E, Gillespie S, Levengood D, Ginty D, Kolodkin A. 1998. Neuropilin-2 is a receptor for semaphorin IV: insight into the structural basis of receptor function and specificity. Neuron 21: 1079-1092.

Giger R, Cloutier J, Sahay A, Prinjha R, Levengood D, Moore SE, Pickering S, Simmons D, Rastan S, Walsh FS, et al. 2000. Neuropilin-2 is required in vivo for selective axon guidance responses to secreted semaphorins. Neuron 25: 29-41.

Helms AW, Johnson JE. 1998. Progenitors of dorsal commissural interneurons are defined by MATH1 expression. Development 125: 919-928.

Huber A, Kania A, Tran T, Gu C, De Marco Garcia N, Lieberam I, Johnson D, Jessell TM, Ginty DD, Kolodkin AL, et al. 2005. Distinct roles for secreted semaphorin signaling in spinal motor axon guidance. Neuron 48: 949-964.

Jaworski A, Long H, Tessier-Lavigne M. 2010. Collaborative and specialized functions of Robol and Robo 2 in spinal commissural axon guidance. J Neurosci 30: 9445-9453.

Jongbloets B, Pasterkamp R. 2014. Semaphorin signaling during development. Development 141: 3292-3297.

Kaprielian Z, Runko E, Imondi R. 2001. Axon guidance at the midline choice point. Dev Dyn 221: 154-181.

Keleman K, Rajagopalan S, Cleppien D, Teis D, Paiha K, Huber LA, Technau GM, Dickson BJ. 2002. Comm sorts robo to control axon guidance at the Drosophila midline. Cell 110: 415-427.

Keleman K, Ribeiro C, Dickson BJ. 2005. Comm function in commissural axon guidance: cell-autonomous sorting of Robo in vivo. Nat Neurosci 8: 156-163.

Kidd T, Brose K, Mitchell KJ, Fetter RD, Tessier-Lavigne M, Goodman CS, Tear G. 1998a. Roundabout controls axon crossing of the CNS midline and defines a novel subfamily of evolutionarily conserved guidance receptors. Cell 92: 205-215.

Kidd T, Russell C, Goodman CS, Tear G. 1998b. Dosage-sensitive and complementary functions of roundabout and commissureless control axon crossing of the CNS midline. Neuron 20: 25-33.

Kolk S, Gunput R, Tran T, van den Heuvel D, Prasad A, Hellemons AJ, Adolfs Y, Ginty DD, Kolodkin AL, Burbach JP, et al. 2009. Semaphorin $3 \mathrm{~F}$ is a bifunctional guidance cue for dopaminergic axons and controls their fasciculation, channel- ing, rostral growth, and intracortical targeting. J Neurosci 29: 12542-12557.

Kolodkin AL, Tessier-Lavigne M. 2011. Mechanisms and molecules of neuronal wiring: a primer. Cold Spring Harb Perspect Biol 3.

Kuwajima T, Yoshida Y, Takegahara N, Petros T, Kumanogoh A, Jessell TM, Sakurai T, Mason C. 2012. Optic chiasm presentation of Semaphorin6D in the context of Plexin-A1 and NrCAM promotes retinal axon midline crossing. Neuron 74: 676-690.

Mann F, Rougon G. 2007. Mechanisms of axon guidance: membrane dynamics and axonal transport in semaphorin signalling. J Neurochem 102: 316-323.

Matei V, Pauley S, Kaing S, Rowitch D, Beisel K, Morris K, Feng F, Jones K, Lee J, Fritzsch B. 2005. Smaller inner ear sensory epithelia in the Neurog 1 null mice are related to earlier hair cell cycle exit. Dev Dyn 234: 633-650.

Nawabi H, Briançon-Marjollet A, Clark C, Sanyas I, Takamatsu H, Okuno T, Kumanogoh A, Bozon M, Takeshima K, Yoshida $\mathrm{Y}$, et al. 2010. A midline switch of receptor processing regulates commissural axon guidance in vertebrates. Genes Dev 24: 396-410.

Park E, Sun X, Nichol P, Saijoh Y, Martin J, Moon A. 2008. System for tamoxifen-inducible expression of cre-recombinase from the Foxa2 locus in mice. Dev Dyn 237: 447-453.

Phelps PE, Alijani A, Tran TS. 1999. Ventrally located commissural neurons express the GABAergic phenotype in developing rat spinal cord. J Comp Neurol 409: 285-298.

Rohm B, Ottemeyer A, Lohrum M, Püschel A. 2000. Plexin/neuropilin complexes mediate repulsion by the axonal guidance signal semaphorin 3A. Mech Dev 93: 95-104.

Sabatier C, Plump AS, Le M, Brose K, Tamada A, Murakami F, Lee EY, Tessier-Lavigne M. 2004. The divergent Robo family protein rig-1/Robo3 is a negative regulator of slit responsiveness required for midline crossing by commissural axons. Cell 117: 157-169.

Stein E, Tessier-Lavigne M. 2001. Hierarchical organization of guidance receptors: silencing of netrin attraction by slit through a Robo/DCC receptor complex. Science 291: 19281938.

Tran TS, Kolodkin A, Bharadwaj R. 2007. Semaphorin regulation of cellular morphology. Annu Rev Cell Dev Biol 23: 263-292.

Tran TS, Rubio ME, Clem RL, Johnson D, Case L, Tessier-Lavigne M, Huganer RL, Ginty DD, Kolodkin AL. 2009. Secreted semaphorins control spine distribution and morphogenesis in the postnatal CNS. Nature 462: 1065-1069.

Tran TS, Carlin E, Lin R, Martinez E, Johnson JE, Kaprielian Z. 2013. Neuropilin2 regulates the guidance of post-crossing spinal commissural axons in a subtype-specific manner. Neural Dev 8: 15 .

Vallstedt A, Kullander K. 2013. Dorsally derived spinal interneurons in locomotor circuits. Ann N Y Acad Sci 1279: 32-42.

Walz A, Rodriguez I, Mombaerts P. 2002. Aberrant sensory innervation of the olfactory bulb in neuropilin-2 mutant mice. I Neurosci 22: 4015-4035.

Wright KM, Lyon KA, Leung H, Leahy DJ, Ma L, Ginty DD. 2012. Dystroglycan organizes axon guidance cue localization and axonal pathfinding. Neuron 76: 931-944.

Xu K, Wu Z, Reinier N, Antipenko A, Tzvetkova-Robev D, Xu Y, Minchenko M, Nardi-Dei V, Rajashankar KR, Himanen J, et al. 2014. Neural migration. Structures of netrin-1 bound to two receptors provide insight into its axon guidance mechanism. Science 344: 1275-1279. 
Hernandez-Enriquez et al.

Yam PT, Kent CB, Morin S, Farmer WT, Alchini R, Lepelletier L, Colman DR, Tessier-Lavigne M, Fournier AE, Charron F. 2012. 14-3-3 proteins regulate a cell-intrinsic switch from sonic hedgehog-mediated commissural axon attraction to repulsion after midline crossing. Neuron 76: 735-749.

Yaron A, Huang P-H, Cheng HJ, Tessier-Lavigne M. 2005. Differential requirement for Plexin-A3 and -A4 in mediating responses of sensory and sympathetic neurons to distinct class 3 semaphorins. Neuron 45: 513-523.
Yoshida Y. 2012. Semaphorin signaling in vertebrate neural circuit assembly. Front Mol Neurosci 6: 71.

Yoshida Y, Han B, Mendelsohn M, Jessell T. 2006. PlexinAl signaling directs the segregation of proprioceptive sensory axons in the developing spinal cord. Neuron 52: 775-788.

Zou Y, Stoeckli E, Chen H, Tessier-Lavigne M. 2000. Squeezing axons out of the gray matter: a role for slit and semaphorin proteins from midline and ventral spinal cord. Cell 102: 363-375. 


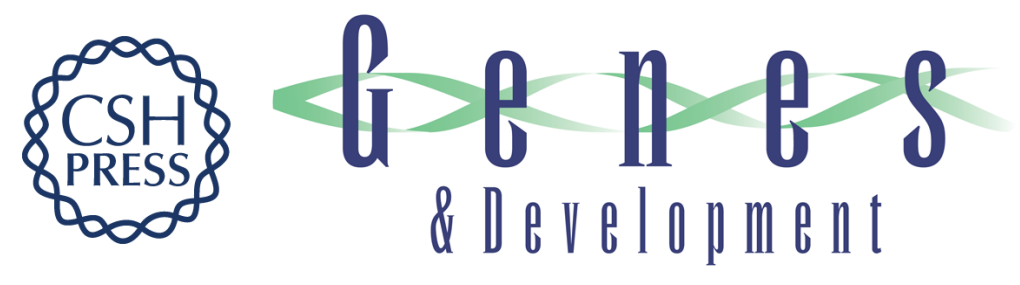

\section{Floor plate-derived neuropilin-2 functions as a secreted semaphorin sink to facilitate commissural axon midline crossing}

Berenice Hernandez-Enriquez, Zhuhao Wu, Edward Martinez, et al.

Genes Dev. 2015, 29:

Access the most recent version at doi:10.1101/gad.268086.115

\section{Supplemental http://genesdev.cshlp.org/content/suppl/2015/12/17/29.24.2617.DC1 \\ Material}

References This article cites 45 articles, 10 of which can be accessed free at:

http://genesdev.cshlp.org/content/29/24/2617.full.html\#ref-list-1

Creative This article is distributed exclusively by Cold Spring Harbor Laboratory Press for the first

Commons six months after the full-issue publication date (see

License http://genesdev.cshlp.org/site/misc/terms.xhtml). After six months, it is available under a Creative Commons License (Attribution-NonCommercial 4.0 International), as described at http://creativecommons.org/licenses/by-nc/4.0/.

Email Alerting Receive free email alerts when new articles cite this article - sign up in the box at the top Service right corner of the article or click here.

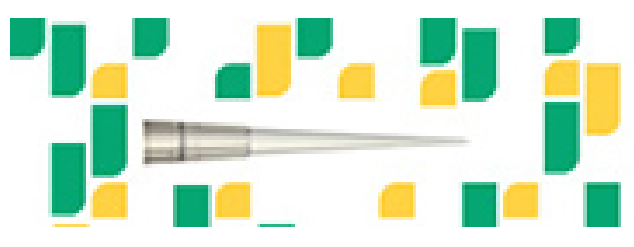

Focused on your science. 